\title{
Structure of Lipid Nanoparticles Containing siRNA or mRNA by Dynamic Nuclear Polarization Enhanced NMR Spectroscopy
}

\author{
Jasmine Viger-Gravel ${ }^{1}$, Anna Schantz ${ }^{2}$, Arthur C. Pinon ${ }^{1}$, Aaron J. Rossini ${ }^{1,3}$, Staffan Schantz ${ }^{4}$, \\ and Lyndon Emsley ${ }^{1 *}$ \\ ${ }^{1}$ Institut des Sciences et Ingénierie Chimiques, Ecole Polytechnique Fédérale de Lausanne (EPFL), CH-1015 \\ Lausanne, Switzerland; ${ }^{2}$ Advanced Drug Delivery, Pharmaceutical Sciences, IMED Biotech Unit, AstraZeneca, \\ Gothenburg, Sweden; ${ }^{3}$ Iowa State University, Department of Chemistry, Ames, IA, USA, ${ }^{4}$ Pharmaceutical \\ Technology \& Development, AstraZeneca, Gothenburg, Sweden.
}

\begin{abstract}
Here we show how dynamic nuclear polarization (DNP) nuclear magnetic resonance (NMR) spectroscopy experiments permit the atomic level structural characterization of loaded and empty lipid nanoparticles (LNPs). The LNPs used here were synthesized by microfluidic mixing technique and are composed of ionizable cationic lipid (DLin-MC3DMA), a phospholipid (DSPC), cholesterol and PEG (DMPE-PEG 2000), as well as encapsulated cargoes which are either phosphorothioated-siRNA (50 or 100\%) or mRNA. We show that LNPs form physically stable complexes with bioactive drug siRNA for a period of 94 days. Relayed DNP experiments are performed to study ${ }^{1} \mathrm{H}-{ }^{1} \mathrm{H}$ spin diffusion and to determine the spatial location of the various components of the LNP by studying the enhancement factors as a function of polarization time. We observe a striking feature of LNPs in the presence and in the absence of encapsulating siRNA or mRNA by comparing our experimental results to numerical spin diffusion modelling. We observe that LNPs form a layered structure and we detect that DSPC and DMPE-PEG 2000 lipids form a surface rich layer in the presence (or absence) of the cargoes, and that the cholesterol and ionizable cationic lipid are embedded in the core. Furthermore, relayed DNP ${ }^{31} \mathrm{P}$ solid-state NMR experiments allow the location of the cargo encapsulated in the LNPs to be determined. Based on the results we propose a new structural model for the LNPs which features a homogeneous core with a tendency for layering of DSPC and DMPE-PEG at the surface.
\end{abstract}

\section{Introduction}

A challenge in medicine today is the ability to deliver drugs to specific targets, for example, to inhibit cancer cells without damaging the surrounding healthy tissues. ${ }^{1}$ Cell membranes are mostly composed of lipid bilayers, and their hydrophobic nature protects the cells from hydrophilic molecules in the extracellular matrix. ${ }^{2-3}$ To achieve cellular internalization of hydrophilic bioactive molecules, many carrier-mediated delivery systems have been proposed. These include for example ionizable cationic lipid DOTMA (N-[1-(2,3-dioley;oxy)propyl]$\mathrm{N}, \mathrm{N}, \mathrm{N}$ - trimethylammonium chloride) and DLinDMA (1,2-dilonoleyloxy-3-dimethylaminopropane) ${ }^{4}$

semiconductor nanocrystals, ${ }^{5}$ carbon nanotubes, ${ }^{6}$ dendrimers, ${ }^{7}$ natural or synthetic polymers, ${ }^{8}$ or cell penetrating peptides. ${ }^{9-10}$ However, most of these systems may show clinical side-effects and have not been approved for in-vivo applications or low efficacy because of poor cellular uptake or clearance by the mononuclear phagocyte system. Lipid nanoparticles (LNPs) show the high promise to overcome these disadvantages for drugdelivery of small interfering RNA (siRNA) or messenger RNA (mRNA) since they can form less toxic highly stable complexes between the cargo and the LNP and achieve target-specific cellular internalization and release of hydrophilic bioactive molecules. ${ }^{2,}$, 11-15 LNPs are spontaneously formed from amphiphilic and hydrophobic constituents to yield complex multicomponent objects known to span a range from 20 to $100 \mathrm{~nm}$ in diameter depending on composition. ${ }^{16}$ The cargo molecules are typically drugs such as siRNA and mRNA. ${ }^{17-18}$ siRNA inhibits endogenous gene expression in mammalian cells and induces degradation of complementary mRNA. ${ }^{17}$ mRNA promotes the right protein expression. ${ }^{19}$ Encapsulation of siRNA or mRNA in LNPs prevents their degradation by nucleases and enables penetration and delivery to the target cells. 

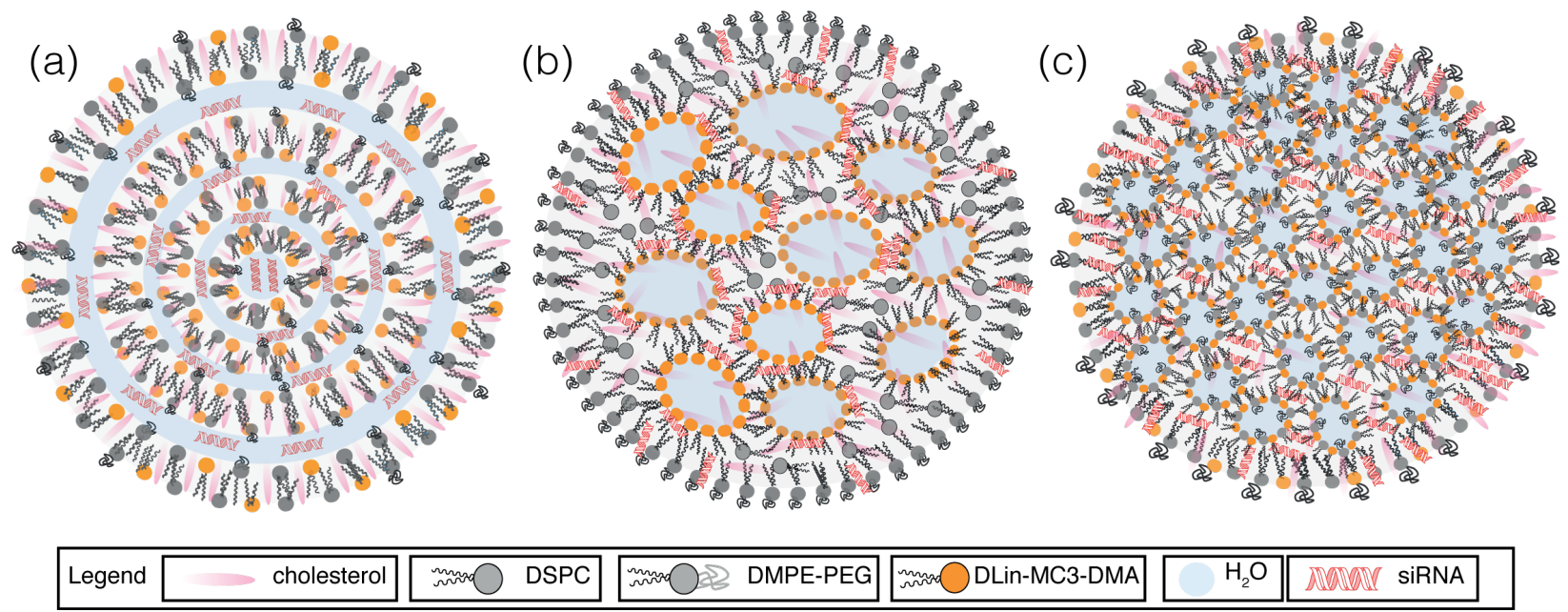

Scheme 1. Cartoon diagrams of three models for LNP structures. (a) Multilamellar vesicles (onion), (b) nanostructure core, and (c) homogeneous core shell

The drug encapsulation and delivery properties can be assumed to be dependent on the exact structure of the LNP, but due to their complexity, there is currently no clear model for LNP structures. Indeed, even the ageing of the particles has not been studied at the molecular level. Furthermore, several models for these selfassembled particles have been proposed in the literature, ${ }^{16}$ including multilamellar vesicles (Scheme 1a), ${ }^{20-22}$ and particles with nanostructured cores (Scheme 1b). ${ }^{23-24}$ However these structures are still in debate. ${ }^{16}$ Here, we use dynamic nuclear polarization (DNP) enhanced NMR spectroscopy to probe the spatial distribution of constituents in LNPs in the presence and absence of siRNA and mRNA, and as a result we propose a new structural model that features a homogeneous core with a tendency toward layering which is different from what is described in literature (Scheme 1C).

Even though there are now multiple studies aimed at characterizing the internalization of a cargo by LNPs using a variety experimental approaches there is still no consensus on the structural organisation of LNPs. To date, the approaches applied to characterize LNPs have included DLS to measure particles sizes, ${ }^{16,25}{ }^{31} \mathrm{P}$ solution NMR to measure encapsulation of siRNA, ${ }^{24}$ cryotransmission electron microscopy (cryo-TEM) to study the overall structure, ${ }^{26}$ or density measurements to probe constitution and molecular modeling to support different structural models. ${ }^{6}$ Small angle X-ray scattering (SAXS) and small angle neutron scattering (SANS) have also been used to characterize the phase behaviour and polymorphism of LNP dispersions, ${ }^{27-28}$ and exchange with the cargo. ${ }^{29}$

In order to probe the detailed structure of the LNPs, one would ideally like to measure the spatial distribution of the different components inside the particles. Traditionally, NMR spectroscopy is the leading method for probing structure and morphology of lipid assemblies. ${ }^{30-31}$ In particular, for LNPs, Leung et al. have successfully observed encapsulation of siRNA using solution ${ }^{31} \mathrm{P}$ NMR experiments. ${ }^{7}$ They observed broadening of the ${ }^{31} \mathrm{P}$ resonances of phosphorothioate siRNA upon encapsulation within the LNP. The broadening of the siRNA ${ }^{31} \mathrm{P}$ NMR signal suggests that upon encapsulation, the siRNA becomes less mobile, indicating that siRNA mainly resides in the lipid phase of the LNP particles rather than in internal water pools, where the siRNA could remain mobile.

It is well established that proton spin-diffusion measurements can be used to determine domain sizes and organisations in a range of materials (substituents) including solid polymer blends, ${ }^{32}$ and this would appear to be a promising approach to determine the domain structures in LNPs. In particular it has recently been shown that spin diffusion of DNP enhanced polarization from a radical containing source phase into a substrate, dubbed relayed-DNP, can be used to determine domain sizes and architectures in complex multi-component mixtures. ${ }^{33-37}$ The approach has been used to determine particle or domain sizes in pharmaceutical formulations, ${ }^{34}$ biomolecules $^{38}$ and microcrystalline solids, ${ }^{33}$ and similar concepts have been used to probe layer dimensions in the supramolecular organization of biosilica. ${ }^{39}$ When sensitivity is high enough, the approach can also be used based on the diffusion of a paramagnetic relaxation enhancement, without the need for DNP. ${ }^{36}$

Here we apply relayed DNP experiments to frozen LNPs in the presence of cryoprotectant to probe the layer structures of LNPs in the presence (or not) of phosphorothioated-siRNA or mRNA. First, we determine that the LNPs show no change in the NMR properties for a period of more than 90 days. Then, we compare our data to predictions for the three models shown in Scheme 1, and find that our DNP results show a layered structure for these $50 \mathrm{~nm}$ complex lipid nanoparticles (Scheme 1c). 
We also probe the location of the cargo, and can distinguish its location between the core, sub-core or surface of the LNP.

\section{Experimental Section}

Synthesis. $\mathrm{D}_{2} \mathrm{O}$-LNP solutions with concentrations of LNP of $60 \mathrm{mg} / \mathrm{mL}$ were manufactured using microfluidic mixing. The nanoparticles were dialyzed overnight in PBS 7.4 in order to remove $\mathrm{EtOH}$ and lower the $\mathrm{pH}$, and then concentrated by ultra-spin filtration. The surrounding $\mathrm{H}_{2} \mathrm{O}$ was exchanged to $\mathrm{D}_{2} \mathrm{O}$ during this process by repeated cycling and equilibration. The LNP samples were prepared with pure $\mathrm{D}_{2} \mathrm{O}$ since partial deuteration of the solvent is usually beneficial for DNP and the components of the LNP were fully protonated. With the high concentration of protonated LNP components, this should make the overall proton concentration similar to $90 \%$ deuterated water-glycerol solutions that typically provide the highest DNP enhancements. All LNP samples are stored in a $4{ }^{\circ} \mathrm{C}$ refrigrator and vortexed or shacked 30 times before use in DNP experiments to prevent aggregation of the LNP.

Dynamic Light Scattering. The mean diameter (ZAverage or number based average) and polydispersity (PDI) of the LNPs was determined using a Zetasizer Nano-ZS (Malvern Instruments Ltd). Dynamic light scattering was performed on diluted samples in $10 \mathrm{mM}$ phosphate buffer at $25^{\circ} \mathrm{C}$ using a refractive index of 1.45 and an absorption of 0.001 for the LNPs. Data was collected at $173^{\circ}$ and the reported diameter is a mean of 3 values. Results are to be found in Supporting information, Table S1.

DNP experiments. All samples were prepared by adding $30 \%$ by volume of cryo-protectant $\left({ }^{12} \mathrm{C}\right.$-glycerol$\left.d_{8}\right)$ to $70 \%$ by volume of LNP sample and $12 \mathrm{mM}$ polarizing agent (AMUPol, MW=726 g/mol). Typically, 20-25 $\mu \mathrm{L}$ of the sample solution described above was transferred using a micro-pipette to a $3.2 \mathrm{~mm}$ o.d. sapphire rotors and capped with a silicon plug. Data were acquired at the Ecole Polytechnique Fédérale de Lausanne using $263 \mathrm{GHz} / 400 \mathrm{MHz}$ Avance I Bruker DNP solid-state NMR spectrometer $\left(v_{\mathrm{L}}\left({ }^{13} \mathrm{C}\right)=100.6\right.$ $\left.\mathrm{MHz}, v_{\mathrm{L}}\left({ }^{31} \mathrm{P}\right)=161.976 \mathrm{MHz}\right)$ equipped with a $3.2 \mathrm{~mm}$ Bruker triple resonance low temperature magic angle spinning (LTMAS) probe and the experiments were performed at ca. $100 \mathrm{~K}$ with a $263 \mathrm{GHz}$ gyrotron capable of outputting a 5-10 W of CW microwaves. The sweep coil of the main magnetic field was set for the $\mu$ wave irradiation occurring at the ${ }^{1} \mathrm{H}$ positive enhancement maximum of the AMUPol biradical. Following this is the usual spectral acquisition for ${ }^{13} \mathrm{C},{ }^{31} \mathrm{P}$, and ${ }^{1} \mathrm{H}$ under CPMAS conditions with spinal64 proton $100 \mathrm{kHz}$ decoupling. Experimental setup and pulse calibration were performed directly on the compound and referenced to the ${ }^{1} \mathrm{H}$ solvent peak following recommendation 10 of P. K. Harris et al. for other nuclei in the solid state. ${ }^{40}$
DNP enhanced ${ }^{31} P$ solid-state NMR. ${ }^{31} \mathrm{P}$ SSNMR experiments used a recycle delay of $1 \mathrm{~s}$ at $9.4 \mathrm{~T}$. The proton $\pi / 2$ pulse length was optimized to $2.50 \mu \mathrm{s}$ providing a $100 \mathrm{kHz}$ proton decoupling. The contact time was $1.4 \mathrm{~ms}$. The spinning frequencies varied between 4 $\mathrm{kHz}$ and $12.5 \mathrm{kHz}$. The CPMAT experiment was acquired un the following conditions: a recycle delay of $1.75 \mathrm{~s}, 336$ scans per increment for a total of 112 at 105 $\mathrm{K}$.

DNP enhanced ${ }^{13} \mathrm{C}$ solid-state NMR. For ${ }^{13} \mathrm{C}$ NMR experiments, the recycle delays were $1 \mathrm{~s}$. The ${ }^{1} \mathrm{H} \pi / 2$ pulse length used for the $\mathrm{CP}$ experiments was $2,5 \mu$ s to afford $100 \mathrm{kHz}{ }^{1} \mathrm{H}$ decoupling. The contact time was typically $20 \mathrm{~ms}$. The MAS frequency used is $10 \mathrm{kHz}$.

Spectra were simulated using WSOLIDS. ${ }^{41}$ DNP enhancements were determined by comparing the integration of the resonance of interest for the spectra acquired with and without $\mu$ wave. Numerical spin diffusion models were constructed with MatLab v7.10. (The MathWorks, Inc.). Additional experimental details may be found in the SI.

\section{Results}

The studies were carried out on LNPs composed of the four constituents shown in Figure 1, an ionizable cationic lipid (DLin-MC3-DMA), a phospholipid (DSPC), cholesterol and a pegylated lipid (DMPE-PEG 2000). As described in detail below, ${ }^{1} \mathrm{H},{ }^{13} \mathrm{C}$ and ${ }^{31} \mathrm{P}$ cross polarization $^{42}$ magic angle spinning ${ }^{43-44}$ (CPMAS) experiments were used to probe the various components of the LNPs which are found to have different chemical shifts $(\delta)$ and DNP enhancements $(\varepsilon) .{ }^{31} \mathrm{P}$ chemical shift anisotropy (CSA) was measured experimentally and interpreted in relation to the electrostatic interaction between LNPs and cargoes, such as siRNA or mRNA. The high sensitivity enhancements offered by DNP enabled the detailed study of frozen LNP solutions with or without encapsulated cargo. Relayed DNP techniques were applied to probe the relative location of the different components in the LNP core, or exterior shell, as well as to probe the position of the cargo. ${ }^{37}$

Initial Characterization. Four samples were studied here: native empty LNP (1); LNP containing 50\% phosphorothioated siRNA strand (2) or at $100 \%$ phosphorothioated (3) and LNP containing mRNA (4). The concentration of each component forming the LNPs is given in Table S2Error! Reference source not found.. The synthesis as well as the encapsulation of the cargo is described in the supporting information together with size distributions of each sample obtained from DLS. 


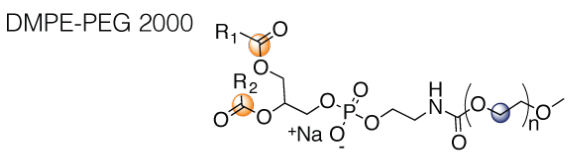

DLin-MC3-DMA

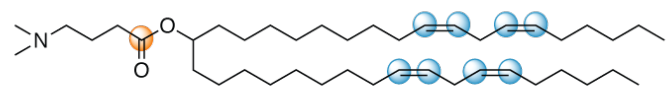

DSPC

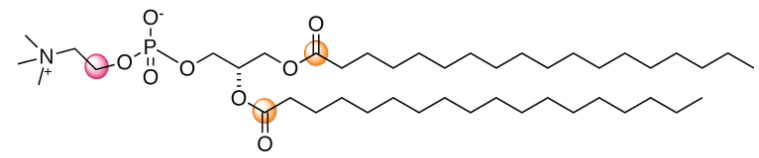

Cholesterol

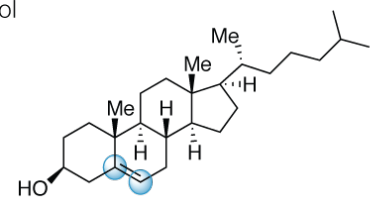

Figure 1. Components of the LNPs studied here. See composition ratios in Table S2 in SI. The functional groups observed for LNPs are resolved in the ${ }^{13} \mathrm{C}$ spectra, and are color coded to the following legend: alkenes ( $\mathrm{R}-\mathrm{HC}=\mathbf{C H}-\mathrm{R})$, ester $\left(\mathrm{R}-\mathrm{CO}_{2}-\mathrm{CH}_{2}-\mathrm{R}\right)$ and $\mathrm{C}_{\alpha}$-phosphate $\left.\mathrm{R}-\mathrm{C}_{\alpha} \mathrm{H}_{2}-\mathrm{PO}_{4}{ }^{3-}\right)$ are in blue, yellow, purple, and pink, respectively.

LNP stability and ageing. Figure 2 shows DNP enhanced ${ }^{13} \mathrm{C}$ CPMAS solid-state NMR spectra of the LNP sample 2 taken over a period of 164 days. Samples were prepared for each time point by mixing an $L N P-D_{2} \mathrm{O}$ $(60 \mathrm{mg} / \mathrm{ml})$ solution with ${ }^{12} \mathrm{C}$-glycerol- $d_{8}$ to form a $70: 30$ $\mathrm{v} / \mathrm{v}$ ratio dispersion and adding AMUPol to afford a concentration of $12 \mathrm{mM}$. The sample was transferred into a sapphire DNP rotor, then frozen inside the pro-cooled DNP probe. The chemical shifts and the observed enhancements at long polarization time are given in Table S5 and S6. Due to the high sensitivity provided by DNP method the natural abundance ${ }^{13} \mathrm{C}$ solid-state NMR spectra can be recorded in minutes, while the microwave off spectra were recorded in about an hour. There is no significant change in the peak positions in the spectra or the enhancements for the first 94 days, illustrating that the formulations are stable over this period. In this procedure, the batch of LNPs were stored at $277 \mathrm{~K}$ and a new DNP sample was prepared for each measurement. A spectrum taken after 164 days shows the same peak positions, but with an enhancement that drops to 11 , this strongly indicates that the components are still intact, but that we see the onset of particle aggregation. Note that in the study of fresh LNP samples, multiple freeze thaw cycles in liquid nitrogen were performed to confirm that the samples are re-dispersible, hence not affected by freezing (not shown).

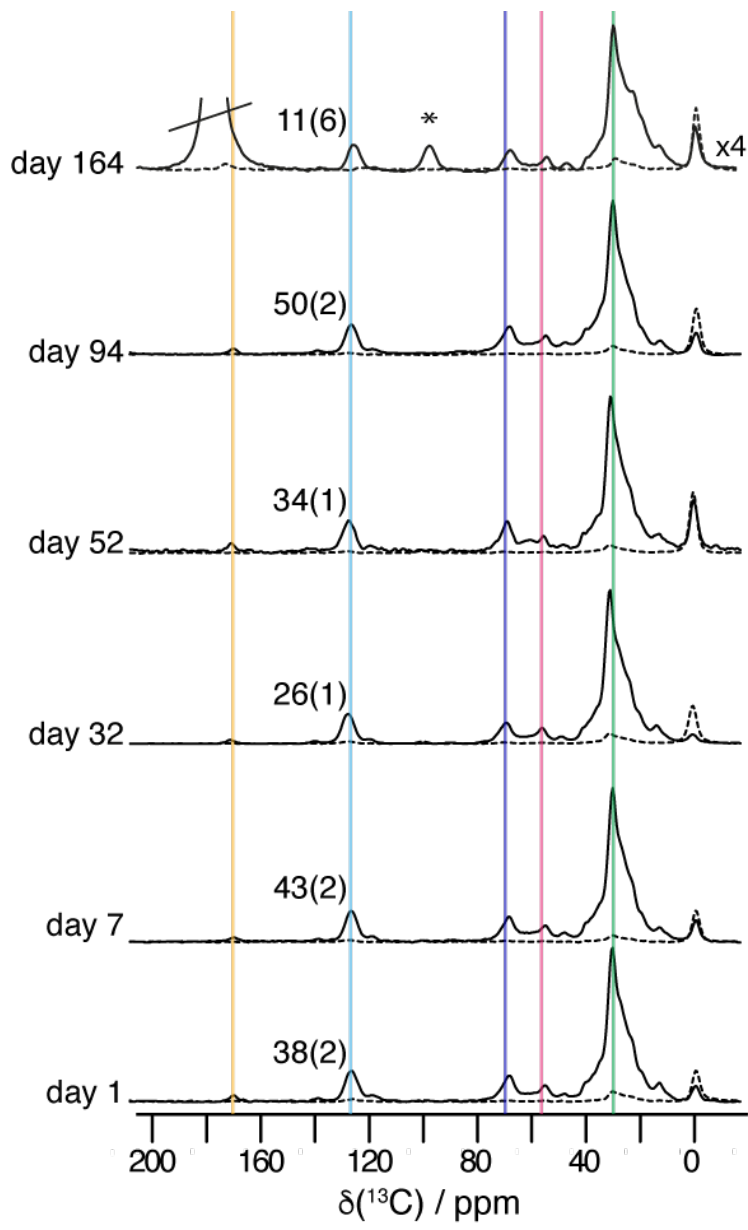

Figure 2. ${ }^{1} \mathrm{H}_{-}{ }^{13} \mathrm{C}$ CPMAS solid-state NMR spectra of sample 2 acquired with a $400 \mathrm{MHz} / 263 \mathrm{GHz}$ DNP system at different times (left) with microwave ( $\mu$ wave) irradiation on (40 mA, black trace) and off (dashed trace). The MAS frequency was between $8 \mathrm{kHz}$ and $12.5 \mathrm{kHz}$ with a temperature ca. at $105 \mathrm{~K}$, and the recycle delay was $1.0 \mathrm{~s}$. The silicone plug is observed in all spectra at a resonance of ca. $0 \mathrm{ppm}$. The components of the LNP are resolved in the ${ }^{13} \mathrm{C}$ spectra, and they are color coded according to the legend described in Figure 1, and the green line corresponds to the $\mathrm{R}-\mathrm{CH}_{2}-\mathrm{R}$ alkyl signal. The vertical line illustrates that the chemical shifts are constant over time. Resonance at 170 ppm corresponds to an external reference $\left({ }^{13} \mathbf{C}=\mathrm{O}\right.$ labelled acetate) used to measure the DNP enhancement of the radical solution (complete details can be found in the SI). The enhancements are noted for the alkene peak at each time point (error in parenthesis).

Spectra of (1) were also recorded, and also showed stability to 94 days. The data for (1) are shown in SI. We note that the different components of the LNPs are resolved in the ${ }^{13} \mathrm{C}$ spectra, with alkane at $30 \mathrm{ppm}, \mathrm{C}_{\alpha^{-}}$ phosphate ( $\left.\mathrm{R}-\mathrm{C}_{\alpha} \mathrm{H}_{2}-\mathrm{PO}_{4}{ }^{3-}-\right)$ at $56 \mathrm{ppm},\left(\mathrm{H}_{3} \mathrm{CO}-\left(\mathrm{CH}_{2}-\right.\right.$ $\left.\mathrm{CH}_{2}-\mathrm{O}\right)_{\mathrm{n}}-\mathrm{R}$ ) ester carbons at $69 \mathrm{ppm}$, alkene at $125 \mathrm{ppm}$ and $\left(\mathrm{R}-\mathrm{CO}_{2}-\mathrm{CH}_{2}-\mathrm{R}\right)$ ester carbons at $170 \mathrm{ppm}$. 
Furthermore, some peaks arise exclusively from particular components of the LNP, such as the alkene resonance $(125 \mathrm{ppm})$ which corresponds to the DLinMC3-DMA cationic lipid and cholesterol, the ester peak at $69 \mathrm{ppm}$ from the DMPE-PEG 2000 and the $\mathrm{C}_{\alpha^{-}}$ phosphate peak at $56 \mathrm{ppm}$ from DSPC, whereas the resonances at $170 \mathrm{ppm}$ and $30 \mathrm{ppm}$ correspond to a superposition of signals from all the LNP components. There are no resolved ${ }^{13} \mathrm{C}$ peaks characteristic of the siRNA or in the 1D spectra of LNP samples containing cargoes, since they overlap with the other constituents. This is further confirmed with a $2 \mathrm{D}{ }^{1} \mathrm{H}-{ }^{13} \mathrm{C}$ HETCOR spectrum where all correlations are attributed to the LNP constituents (see SI, Figure S4).

DNP Enhanced ${ }^{31} P$ Solid-State NMR. NMR signals from both RNA and the phosphate groups of the LNPs are observed in DNP enhanced ${ }^{31} \mathrm{P}$ solid-state NMR spectra (Figure 3). The chemical shift of the phosphate $\left(\mathrm{PO}_{4}{ }^{3-}\right)$ functional group of DSPC and DMPE-PEG components are observed at $10 \mathrm{ppm}$ in all systems, in agreement with the literature values. ${ }^{45-48}$ The phosphorothioate group of the siRNA $\left(\mathrm{S}=\mathrm{PO}_{3}{ }^{2-}\right)$ resonates at an isotropic chemical shift of $67 \mathrm{ppm}$ which permits differentiation from the $\mathrm{PO}_{4}{ }^{3-}$ functional groups of the LNP components. Figure 3d compares the DNP enhanced ${ }^{31} \mathrm{P}$ CPMAS spectra of siRNA which is $100 \%$ phosphorothioated dissolved in $\mathrm{D}_{2} \mathrm{O}$ and the ${ }^{31} \mathrm{P}$ CPMAS spectra of samples $\mathbf{1}, \mathbf{3}$, and $\mathbf{4}$.

Table 1. Experimental ${ }^{31} \mathrm{P}$ CS tensor parameters. ${ }^{a}$

\begin{tabular}{|c|c|c|c|c|}
\hline sample & & $\begin{array}{l}\delta_{\text {iso }} / \\
\text { ppm }\end{array}$ & $\mathrm{ppm}^{\mathrm{b}}$ & $\kappa^{b}$ \\
\hline 1 & $\begin{array}{l}\mathrm{PO}_{4}{ }^{3-} \\
\mathrm{LNP}\end{array}$ & $10(4)$ & $183(5)$ & $0.33(0.04)$ \\
\hline \multirow[t]{2}{*}{3} & $\begin{array}{l}\mathrm{PO}_{4}{ }^{3-} \\
\mathrm{LNP}\end{array}$ & $7(5)$ & $182(5)$ & $0.35(0.02)$ \\
\hline & $\begin{array}{l}\mathrm{S}=\mathrm{PO}_{3}{ }^{2-} \\
\text { siRNA }\end{array}$ & $65(3)$ & $200(12)$ & $0.54(0.03)$ \\
\hline $\operatorname{siRNA} / \mathrm{D}_{2} \mathrm{O}$ & $\begin{array}{l}\mathrm{S}=\mathrm{PO}_{3}{ }^{2-} \\
\text { siRNA }\end{array}$ & $67(4)$ & $196(16)$ & $0.39(0.03)$ \\
\hline 4 & $\begin{array}{l}\mathrm{PO}_{4}{ }^{3-} \\
\mathrm{LNP}\end{array}$ & $7(5)$ & $183(5)$ & $0.31(0.02)$ \\
\hline
\end{tabular}

${ }^{a}$ Error bounds are given in parentheses. ${ }^{b}$ Isotropic chemical shift: $\delta_{\text {iso }}=\left(\delta_{11}+\delta_{22}+\delta_{33}\right) / 3$; span: $\Omega \approx \delta_{11}-\delta_{33}$; skew: $\kappa=3\left(\delta_{22}-\delta_{\text {iso }}\right) /\left(\delta_{11}-\delta_{33}\right)$, where $\delta_{11} \geq \delta_{22} \geq \delta_{33}$.

The ${ }^{31} \mathrm{P}$ CPMAS spectra were acquired with a $4.5 \mathrm{kHz}$ MAS frequency in order to generate spinning sideband manifolds that can be fit to determine the ${ }^{31} \mathrm{P}$ chemical shift tensors. It should be noted that for compound $3 \mathrm{a}^{31} \mathrm{P}$ CPMAT experiment was acquired in order to separate the chemical shift anisotropy (CSA) from the isotropic part, hence a slice of the $2 \mathrm{D}$ experiment provides a slow MAS spectrum. This type of experiment is ideal to resolve different sites and extract CSA information, which permits simulation of the chemical shift tensor without ambiguity (see SI for more details). The ${ }^{31} \mathrm{P}$ chemical shift tensors extracted from these spectra are given in Table 1.

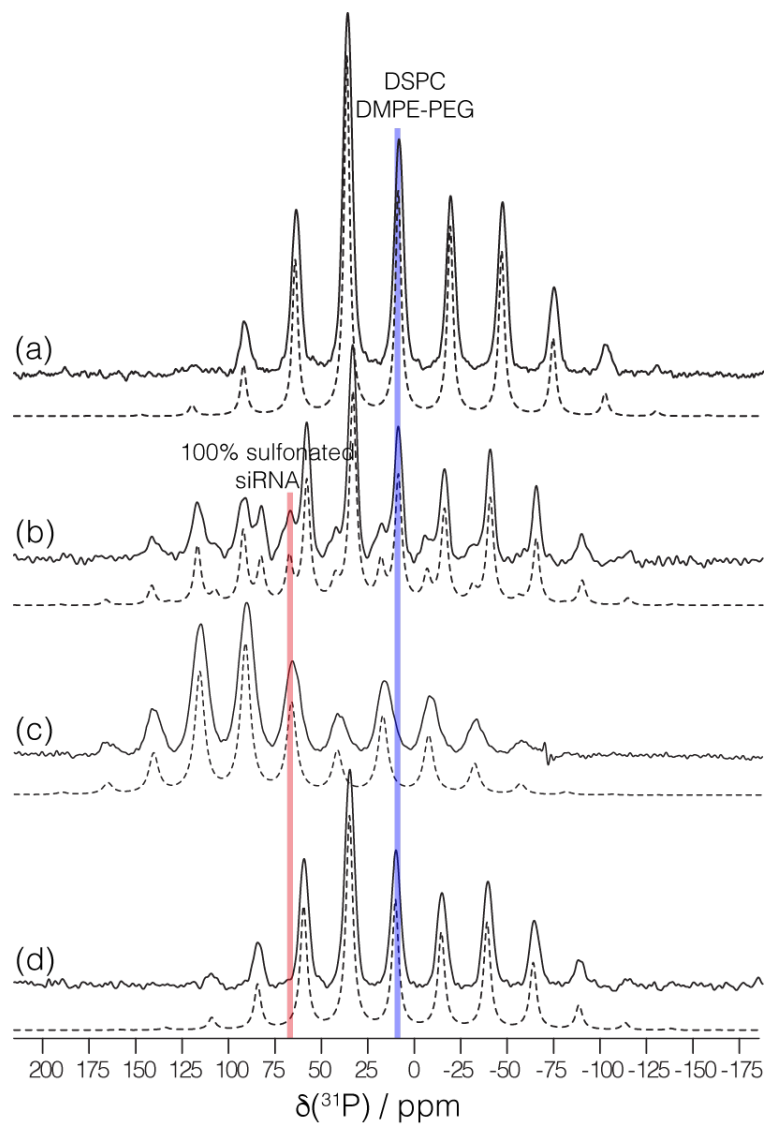

Figure 3. Experimental ${ }^{31}$ P CPMAS DNP spectra (solid line) and simulated spectra (dashed lines) acquired with $\mu$ wave on and a slow spinning speed (4.5 kHz) for sample (a) 4, (b) 3 (100\% phosphorothioated cargo) and (c) $100 \%$ phosphorothioated siRNA dispersed in $\mathrm{D}_{2} \mathrm{O}$ and (d) $\mathbf{1}$. The centerbands are highlighted in blue at $10 \mathrm{ppm}$ for the $\mathrm{PO}_{4}{ }^{3-}$ group of the lipids, and $\mathrm{S}=\mathrm{PO}_{3}{ }^{2-}$ group of the cargo in red at $68 \mathrm{ppm}$.

Previous MD simulations and solution ${ }^{31} \mathrm{P}$ experiments suggests that the cargo interacts through electrostatic interactions with the LNP, and more specifically through a RNA-cationic lipid interaction. ${ }^{23}$ Hence, we expect to observe a change in the chemical shift (CS) tensor for the phosphorothioate group of the siRNA when encapsulated in the LNP if electrostatic interactions are present between the LNP and siRNA. It has previously been shown that the interaction of metal ions $\left(\mathrm{Mg}^{2+}, \mathrm{Li}^{2+}, \mathrm{Cd}^{2+}\right.$, $\mathrm{Na}^{2+}$ ) with phosphate groups in DNA or nucleic acids induces about $5 \mathrm{ppm}$ change in the span $(\Omega)$ and $0-10$ ppm change in isotropic chemical shift due to electrostatic interactions. ${ }^{46-50}$ The CS tensor values in Table 1 for the cargo components $\left(\mathrm{S}=\mathrm{PO}_{3}{ }^{2-}\right)$ are found here to be the same within error, in the presence of LNPs or not. Hence, if the siRNA is interacting at all with the cations then the interactions are weak and might not be 
observable by NMR. Also, as expected, the CS tensors of the lipid are the same in the presence or not of siRNA.

Relayed-DNP Measurements. We used relayed-DNP ${ }^{34}$,

${ }^{37}$ to probe the morphology of the LNPs by estimating the relative position of the different LNP constituents with respect to the LNP surface (i.e., surface or core). Relatively higher DNP enhancements are expected for components located at the surface as they are easily hyperpolarized due to their proximity to the radical solution, and lower $\varepsilon$ values will be observed for groups buried inside the LNP core, because polarization is lost during diffusion of polarization from the surface to the inside of the LNP domain. ${ }^{33-34,39}$

In the LNP systems $\mathbf{1}, \mathbf{2}, \mathbf{3}$ and $\mathbf{4}$ the enhancement values obtained are relatively small for the LNP components $(\varepsilon \sim 21-55)$ when compared to the enhancement factor of the radical solution $(\varepsilon \approx 100$, see SI for complete details on measurements). This difference in the enhancement value is a clear indication that the LNPs are a heterogeneous system polarized through ${ }^{1} \mathrm{H}-{ }^{1} \mathrm{H}$ spin diffusion ${ }^{37}$ from the radical containing solution located outside the LNP domains.

The experimentally observed ${ }^{13} \mathrm{C}$ and ${ }^{31} \mathrm{P}$ signal buildup curves as a function of the polarization time are shown for empty and loaded LNPs in the supporting information. The build-up curves were fit with stretched exponential functions (see Equation 1) to the values given in Tables S8-S12. The observed stretching parameters $\beta$ are different from 1 , which is also consistent with a model of relayed polarization transfer into the particle. ${ }^{33-34}$

Figures S6-S15 (see SI) shows the measured $\varepsilon$ for ${ }^{13} \mathrm{C}$ and ${ }^{31} \mathrm{P}$ resonances of the different components as a function of polarization delay for LNP samples 1, 2, 3, and 4. Apart from the first point, the enhancement is found to be constant as a function of polarization delay for each component, which is consistent with the expected (small) size of the LNPs $(\sim 50 \mathrm{~nm})$, which means that they are polarized very rapidly.

\section{Discussion}

Proposed model structures for the lipid nanoparticles. No conclusion on atomic level organisation of LNPs has been reached yet. We have considered the following three possible models (see Scheme 1), as follows.

Multilamellar vesicle model. ${ }^{21,26}$ The multilamellar vesicle model includes concentric layered rings such that the exterior surface is formed of DMPE-PEG, cholesterol, DSPC and DLin-MC3-DMA. Within this exterior shell is a layer of water containing the cargo. The bilayer of lipids is then repeated, alternating with the water/cargo layer.

Nanostructured core model. ${ }^{23-24}$ The nanostructured core model presents a hydrophobic core with a surface monolayer formed of a polyethylene glycol lipid. The hydrophobic core is formed of inverted cationic lipids that are separated by asymmetric water pores formed during LNP synthesis, along with homogeneously dispersed cholesterol and DSPC. Computer modeling suggests that no cargo such as siRNA is present inside the water cavities and predicts that the cargo interacts with the cationic lipid interface as well as with the lipids at the inner surface. $^{23}$

Homogeneous core shell. This model has the surfactant components (DMPE-PEG, DSPC, DLin-MC3-DMA, and cholesterol) homogeneously dispersed throughout the particle, with a thin shell, and the interior being made up of small evenly dispersed inverted micelles throughout the domain. The inverted micelles form small water pockets. The cargo is homogeneously dispersed throughout the structure.

The multilamellar vesicle model to describe the structure of cargo/LNP systems has been ruled out by cryo-TEM, density measurements and molecular modeling. ${ }^{24}$ Cryo-TEM demonstrates the presence of hydrophobic cores inside the LNP, because the electron density increases when compared to the liposome system. $^{24,51}$

Modeling of Relayed DNP. The small measured enhancement values $(\varepsilon \sim 21-55, \mathrm{SI})$ at long polarization times and rapid relaxation build up times with microwave irradiation on $\left(\left(T_{\mathrm{B}, \text { on }}\right) \sim 1-2.5 \mathrm{~s}\right.$ are consistent with particle diameters of less than $100 \mathrm{~nm}(\sim 36-53 \mathrm{~nm}, \mathrm{SI})$.

Two models which represent the possible extreme cases of the LNP structure, homogeneous or layered domain, are compared by numerical proton spin diffusion simulations. The structures are shown in Scheme 2.
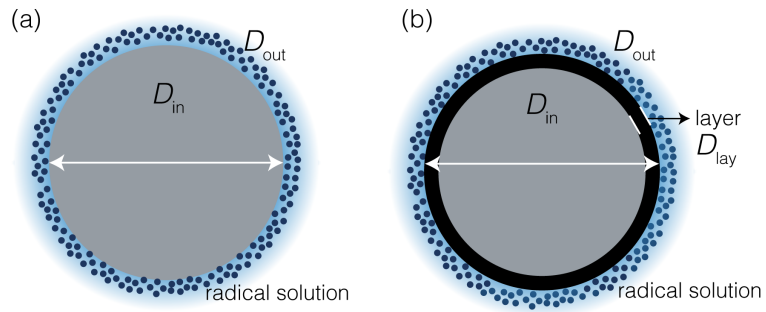

Scheme 2. Scheme of models used to simulate numerically the proton spin diffusion behavior observed in the various LNP systems, where in (a) model I has a homogenous domain (b) model II adds a layer at the surface of the domain. The symbols $D_{\text {in }}, D_{\text {lay }}$ and $D_{\text {out }}$ correspond to the different spin diffusion coefficients inside the LNP domain, layer and bulk radical solution, respectively.

Figure 4 compares the best fits between the experimentally observed averaged enhancement $(\vec{\varepsilon})$ and the predictions of the numerical simulations for Model I and II. Note that for the simulations, we consider polydispersity of the LNP size, as measured by DLS. We also assume that the spin diffusion constants $(D)$ inside the LNP and in the LNP layer (in the case of model II) are lower $\left(D_{\text {in }} \sim 10^{-4} \mu \mathrm{m}^{2} \mathrm{~s}^{-1}\right)$ than outside in the radical solution $\left(D_{\text {out }}=1 \cdot 10^{-3} \mu \mathrm{m}^{2} \mathrm{~s}^{-1}\right)$. The lower spin diffusion 
constant inside the LNP is included to account for molecular dynamics which might be explained by additional mobile moieties such as methyl groups and alkane chains. ${ }^{37-38}$ Model I mimics a homogeneous core model which displays an equal value for the enhancement for all components. Model II represents a layered structure where the simulated polarization for the surface, core and global regions correspond to the LNP components: DSPC and DMPE-PEG, cholesterol and DLin-MC3-DMA, and the alkanes, respectively. Further details of the numerical models and the parameters used are given in the SI.

We immediately see that for Model I, which corresponds to the homogeneous core structure where all LNP components are uniformly distributed in the domain, the simulations predict the same value of the enhancement for all the different components. This is clearly not in agreement with our data. In contrast, the simulations for Model II are in good agreement with the data and predict that we should see different values of the enhancements for the different components, with surface (layer) $>$ global (average of layer and core) $>$ core. As mentioned above, higher enhancements are expected for components localised closer to the polarization source at the surface. DSPC and DMPE-PEG have higher enhancements than the other components in all four LNP samples. This suggests there is an enrichment of DSPC and DMPE-PEG lipids at the surface of the particles, and

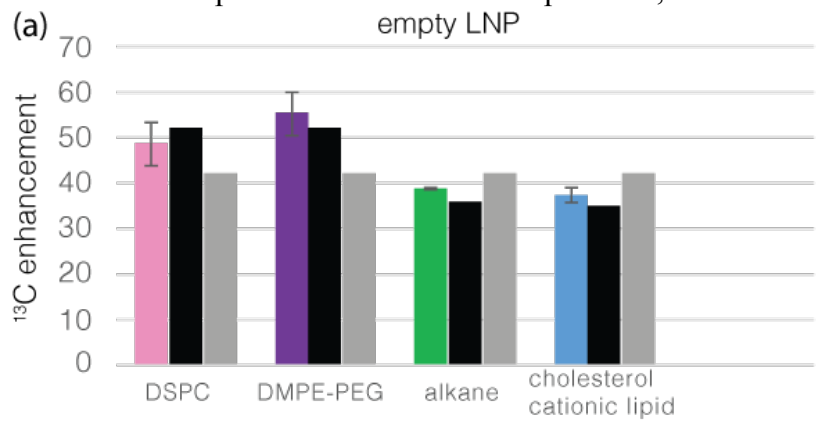

(b)

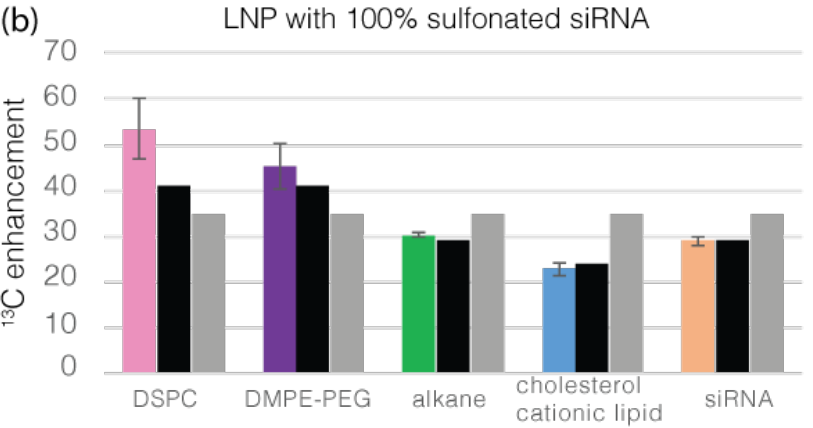

that DLin-MC3-DMA and cholesterol are located in the core region of the LNP domain.

It is evident that the LNP size increases in going from empty LNPs to LNPs containing the most hydrophilic cargo (see SI) due to encapsulation of a hydrophilic cargo. This could occur due to an increase in internal water content that in turn results in an increase in LNP size as shown schematically in Figure 5. As the water content increases inside the LNP, the amphiphilic DMPE-PEG should no longer be as concentrated at the surface, but may be distributed further into the LNP due to the presence of an increased internal water interface. As the DSPC is hydrophobic in nature, the increasing amount of internal water may lead it to become relatively enriched in the surface lipid layer. Numerical calculations show that as the number of water molecules increases inside the LNP there is a relative decrease in the volume not occupied by water inside the LNPs (see SI for details of the calculations). That volume corresponds to the space were DSPC is located. This might be supported by the observed change in DNP enhancement values of the DSPC and DMPE-PEG components in samples $\mathbf{2}$ and $\mathbf{3}$ in Figure 4. However, it is not observed for sample 4, and the changes are at the limit of our signal to noise ratios, so may not be significant. This will be investigated further in future work.
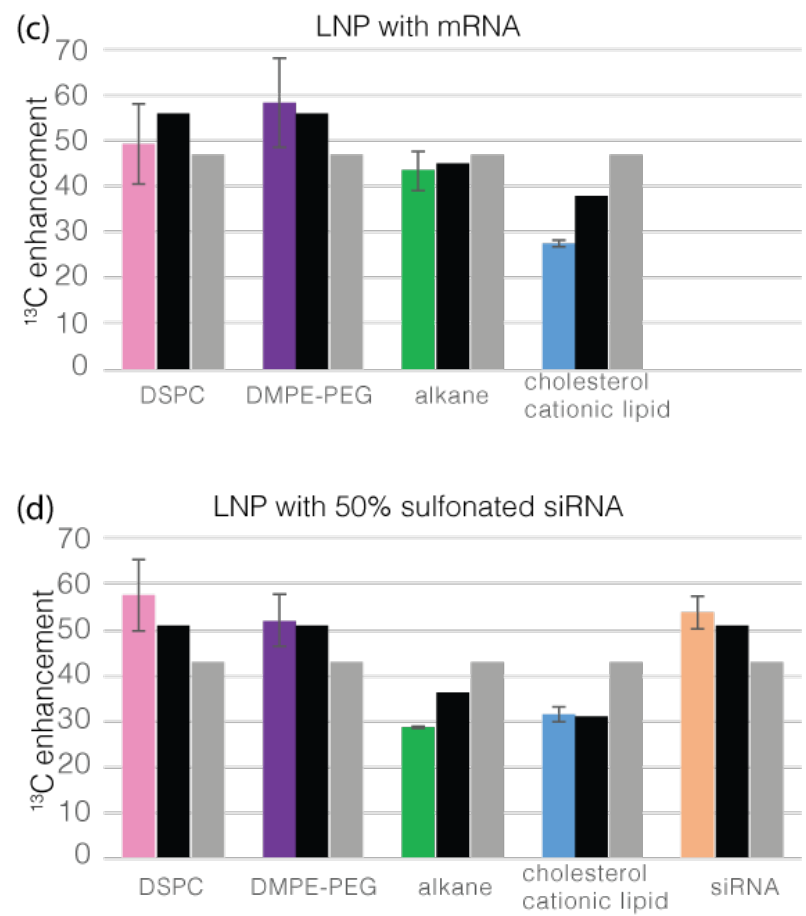

Figure 4. Histogram of experimentally measured average enhancement ( $\bar{\varepsilon}$ : average of 8 longest polarization delays) of the different LNP components DSPC (pink), DMPE-PEG 2000 (purple), alkane (green) and cholesterol as well as cationic lipid (blue) for samples 1 (a), 3 (b), 2 (d) and 4 (c). Respective calculated enhancements values for each LNP component from numerical spin diffusion for Model I (gray) and Model II (black) are shown for comparison. 


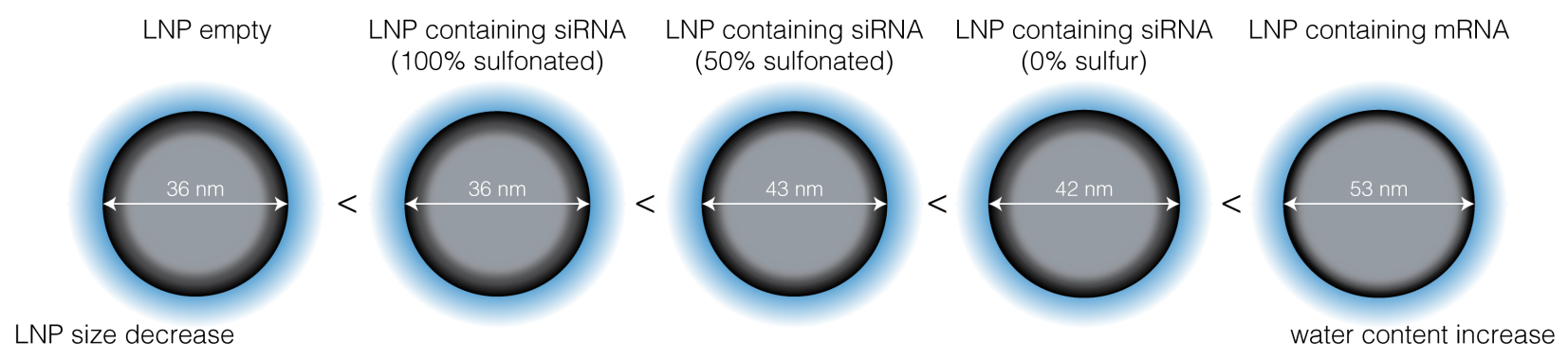

Figure 5. Scheme illustrating the increase in number average size of the LNP as a function of water content which may be dependent on the hydrophilicity of the cargo encapsulated, where blue illustrates bulk radical solution, gray core LNP and black surface layer.

Finally, as regards the cargo, the ${ }^{31} \mathrm{P}$ experimental enhancement values (Figure S16) of 29 and 54 for LNP containing $100 \%$ and $50 \%$ phosphorothioated siRNA, respectively. The ${ }^{13} \mathrm{C}$ and ${ }^{31} \mathrm{P}$ DNP enhancements are directly comparable because in both cases $\mathrm{CP}$ is used to transfer DNP enhanced ${ }^{1} \mathrm{H}$ polarization to the observed nuclei. As can be seen in the SI, the average enhancement measured for the $\mathrm{PO}_{4}{ }^{3-}$ resonance (corresponding to DSPC and DMPE-PEG) is the same as the average for the ${ }^{13} \mathrm{C}$ resonances in DSPC and DMPE-PEG. Hence, the numerical spin diffusion simulations using Model II for the siRNA cargoes might provide insight on the localization inside the LNP domain. As described above, the simulations suggest that for sample $\mathbf{3}$, the cargo is situated inside the sub-core and for sample $\mathbf{2}$ it is located in the surface layer (see Figure 4). This result is in line with the relative hydrophilicity of siRNA and mRNA.

\section{Conclusions}

To our knowledge, this study represents the first systematic experimental examination of LNPs in the solid-state using relayed-DNP methods, a powerful characterization tool, to study the stability as well as to elucidate their structure at the atomic level and nanoscale. The high sensitivity of DNP enhanced NMR spectroscopy permits acquisition of ${ }^{13} \mathrm{C}$ and ${ }^{31} \mathrm{P}$ solidstate NMR spectra in minutes. Measurements of the DNP enhancement factor provides an additional parameter to study stability of LNPs and probe the location of the different components. LNPs in the presence and in the absence of the bioactive drugs siRNA and mRNA have been prepared and characterized by ${ }^{31} \mathrm{P}$, and ${ }^{13} \mathrm{C}$ relayed DNP experiments. We observe that LNPs form physically stable complexes with their cargo by monitoring that the ${ }^{13} \mathrm{C}$ values as well as the enhancement factors are constant over a 94-day period.

Phosphorus and carbon chemical shifts are good probes to distinguish between the various constituents of the LNP and determine their morphology by estimating the relative position of each components. Higher DNP enhancements are expected for LNP components located at the surface due to their proximity to the biradical then components buried inside the domain. The LNPs in this study are $\sim 50 \mathrm{~nm}$ in diameter size and are polarized instantly, hence the observed ${ }^{13} \mathrm{C}$ and ${ }^{31} \mathrm{P}$ enhancements profiles for all LNP components form a plateau over long polarization delays. The enhancement values as a function of polarization delays are interpreted using two types of numerical spin diffusion models: (i) homogeneous core and (ii) layer model to elucidate the structure of LNPs. The experimental data agreed best with the layer model which predicts an LNP structure enriched of both DMPE-PEG 2000 and DSPC at the surface and a dense core composed of the cationic lipid and cholesterol. This is also the first evidence of the structure of LNPs forming a DSPC and DMPE-PEG 2000 lipid rich surface layer in the presence or absence of cargoes.

${ }^{31} \mathrm{P}$ CSA values of the $\mathrm{PO}_{4}{ }^{3-}$ groups of either the LNP or siRNA are independent of their environment, which demonstrates that electrostatic interactions between siRNA and LNPs are weak for these types of system. The $\varepsilon\left({ }^{31} \mathrm{P}\right)$ values as a function of polarization delay of the cargoes can be interpreted using spin diffusion model in order to locate its position in the LNP domain. In that context, we observe that for sample $\mathbf{3}$, the cargo is located in the sub-core of the LNP, and for sample $\mathbf{2}$ it is located at the surface.

In the future, elucidating the core structure will provide a better understanding of the mechanism of encapsulation of the cargo by the LNP, assisting in the and to rational design of future LNPs for selective cell targeting as well as optimization of future drug delivery LNP systems. Furthermore, the DNP enhanced NMR spectroscopy methods demonstrated here should be applicable for locating bioactive drugs in other advanced drug delivery systems.

\section{ASSOCIATED CONTENT}

Supporting Information. Additional experimental details; particle size measurement on Zetasizer and PDI; additional DNP SENS spectra and acquisition information, Build-up curves and diffusion model simulations. The following files are available free of charge (i.e., PDF).

\section{AUTHOR INFORMATION}




\section{Corresponding Author}

Ecole Polytechnique Fédérale de Lausanne, Institut des sciences et ingénirie chimiques, BCH 1530 (Batochime), Lausanne, Switzerland 1004, Tel.: +41 (0) 02169393 86; Fax: +41 (0)21 $693 \quad 98$ 95, E-mail: lyndon.emsley@epfl.ch

\section{Author Contributions}

The manuscript was written through contributions of all authors. All authors have given approval to the final version of the manuscript.

Funding Sources

Financial support from ERC Advanced Grant No. 320860 and AsttraZeneca.

\section{ACKNOWLEDGMENT}

Financial support from AstraZeneca and ERC Advanced Grant No. 320860 is acknowledged for this work. We would like to thank Marianna Yanez Arteta, Aleksandra Dabkowska and Lennart Lindfors for sharing SANS and SAXS data acquired prior to publication and Lennart Lindfors for the mRNA sample (AstraZeneca). Gunilla Nilsson and Svante Johansson (AstraZeneca) are thanked for quantitative analyses of the formulations.

\section{ABBREVIATIONS}

LNP, lipid nanoparticle; siRNA, mRNA small interfering ribonuclease; NMR, nuclear magnetic resonance; DNP SENS, dynamic nuclear polarization surface enhanced spectroscopy; CPMAS, cross polarization magic angle spinning; CSA, chemical shift anisotropy; HETCOR, heteronuclear correlation; $T_{\mathrm{B}}$, build-up time; $\mu$ wave, microwave.

\section{REFERENCES}

1. Kanasty, R.; Dorkin, J. R.; Vegas, A.; Anderson, D., Delivery Materials for siRNA Therapeutics. Nat. Mater. 2013, 12, 967-977.

2. Allen, T. M.; Cullis, P. R., Liposomal Drug Delivery Systems: From Concept to Clinical Applications. Adv. Drug. Deliv. Rev. 2013, 65, 36-48.

3. Zhigaltsev, I. V.; Maurer, N.; Edwards, K.; Karlsson, G.; Cullis, P. R., Formation of DrugArylsulfonate Complexes inside Liposomes: A Novel Approach to Improve Drug Retention. J. Control. Release 2006, 110, 378-386.

4. Semple, S. C., et al., Rational Design of Cationic Lipids for siRNA Delivery. Nat. Biotechnol. 2010, 28, 172-178.

5. Michalet, X.; Pinaud, F. F.; Bentolila, L. A.; Tsay, J. M.; Doose, S.; J. J. Li; Sundaresan, G.; Wu, A. M.; Gambhir, S. S.; Weiss, S., Quantum Dots for Live Cells, in Vivo Imaging, and Diagnostics. Science 2005, 307, 538-544.
6. Liu, Z.; Winters, M.; Holodniy, M.; Dai, H., siRNA Delivery into Human T Cells and Primary Cells with Carbon-Nanotube Transporters. Angew. Chem. Int. Ed. 2007, 46, 2023-2027.

7. Menjoge, A. R.; Kannan, R. M.; Tomalia, D. A., Dendrimer-Based Drug and Imaging Conjugates: Design Considerations for Nanomedical Applications. Drug Discov. Today 2010, 15, 171-185.

8. Putnam, D., Polymers for Gene Delivery across Length Scales. Nat. Mater. 2006, 5, 439-451.

9. Marchetti, A.; Chen, J.; Pang, Z.; Li, S.; Ling, D.; Deng, F.; Kong, X., Understanding Surface and Interfacial Chemistry in Functional Nanomaterials Via Solid-State NMR. Adv. Mater. 2017, 2-38.

10. Xiong, X.-B.; Lavasanifar, A., Traceable Multifunctional Micellar Nanocarriers for CancerTargeted Co-Delivery of Mdr-1 siRNA and Doxorubicin. ACS Nano 2011, 5, 5202-5213.

11. Tam, Y. Y.; Chen, S.; Cullis, P. R., Advances in Lipid Nanoparticles for siRNA Delivery. Pharmaceutics 2013, 5, 498-507.

12. Huang, L.; Liu, Y., In Vivo Delivery of RNAi with Lipid-Based Nanoparticles. Annu. Rev. Biomed. Eng. 2011, 13, 507-530.

13. Malam, Y.; Loizidou, M.; Seifalian, A. M., Liposomes and Nanoparticles: Nanosized Vehicles for Drug Delivery in Cancer. Trends Pharmacol. Sci. 2009, 30, 592-599.

14. Stewart, M. P.; Sharei, A.; Ding, X.; Sahay, G.; Langer, R.; Jensen, K. F., In Vitro and Ex Vivo Strategies for Intracellular Delivery. Nature 2016, 538, 183-192.

15. Wittrup, A.; Lieberman, J., Knocking Down Disease: A Progress Report on siRNA Therapeutics. Nat. Rev. Genet. 2015, 16, 543-552.

16. Pabst, G.; Kucerka, N.; Nieh, M.-P.; Katsaras, J., Liposomes, Lipid Bilayers and Model Membranes from Basic Research to Application. CRC PRESS 2014, 291-316.

17. Zimmermann, T. S., et al., Rnai-Mediated Gene Silencing in Non-Human Primates. Nature 2006, 441, 111-114.

18. Leung, A. K.; Tam, Y. Y.; Cullis, P. R., Lipid Nanoparticles for Short Interfering RNA Delivery. $A d v$. Genet. 2014, 88, 71-110.

19. Kauffman, K. J.; Dorkin, J. R.; Yang, J. H.; Heartlein, M. W.; DeRosa, F.; Mir, F. F.; Fenton, O. S.; Anderson, D. G., Optimization of Lipid Nanoparticle Formulations for mRNA Delivery in Vivo with Fractional Factorial and Definitive Screening Designs. Nano Lett. 2015, 15, 7300-6.

20. Huan, C.-H., Studies on Phosphatidylcholine Vesicles. Formation and Physical Characteristics. Biochemistry 1969, 8, 344-352. 
21. Jeffs, L. B.; Palmer, L. R.; Ambegia, E. G.; Giesbrecht, C.; Ewanick, S.; MacLachlan, I., A Scalable, Extrusion-Free Method for Efficient Liposomal Encapsulation of Plasmid DNA. Pharm. Res. 2005, 22, 362-372.

22. Maurer, N.; Wong, K. F.; Stark, H.; Louie, L.; Mcintosh, D.; Wong, T.; Scherrer, P.; Semple, S. C.; Cullis, P. R., Spontaneous Entrapment of Polynucleotides Upon Electrostatic Interaction with Ethanol-Destabilized Cationic Liposomes. Biophys. J. 2001, 80, 2310-2326.

23. Rozmanov, D.; Baoukina, S.; Tieleman, D. P., Density Based Visualization for Molecular Simulation. Faraday Discuss. 2014, 169, 225-243.

24. Leung, A. K.; Hafez, I. M.; Baoukina, S.; Belliveau, N. M.; Zhigaltsev, I. V.; Afshinmanesh, E.; Tieleman, D. P.; Hansen, C. L.; Hope, M. J.; Cullis, P. R., Lipid Nanoparticles Containing Sirna Synthesized by Microfluidic Mixing Exhibit an Electron-Dense Nanostructured Core. J. Phys. Chem. C 2012, 116, 18440-18450.

25. Chen, S.; Tam, Y. Y.; Lin, P. J.; Sung, M. M.; Tam, Y. K.; Cullis, P. R., Influence of Particle Size on the in Vivo Potency of Lipid Nanoparticle Formulations of siRNA. J. Control. Release 2016, 235, 236-244.

26. Crawford, R.; Dogdas, B.; Keough, E.; Haas, R. M.; Wepukhulu, W.; Krotzer, S.; Burke, P. A.; SteppLorenzino, L.; Bagchi, A.; Howell, B. J., Analysis of Lipid Nanoparticles by Cryo-EM for Characterizing siRNA Delivery Vehicles. Int. J. Pharm. 2011, 403, 237244.

27. Bunjes, H.; Unruh, T., Characterization of Lipid Nanoparticles by Differential Scanning Calorimetry, XRay and Neutron Scattering. Adv. Drug. Deliv. Rev. 2007, 59, 379-402.

28. Zhang, J.; Fan, H.; Levorse, D. A.; Crocker, L. S., Interaction of Cholesterol-Conjugated Ionizable Amino Lipids with Biomembranes: Lipid Polymorphism, Structure-Activity Relationship, and Implications for siRNA Delivery. Langmuir 2011, 27, 9473-9483.

29. Tajik-Ahmadabad, B.; Mechler, A.; Muir, B. W.; McLean, K.; Hinton, T. M.; Separovic, F.; Polyzos, A., A QCM-D and SAXS Study of the Interaction of Functionalised Lyotropic Liquid Crystalline Lipid Nanoparticles with siRNA. Chembiochem 2017, 18, 921930 .

30. Rydall, J. R.; Macdonald, P. M., Investigation of Anion Binding to Neutral Lipid Membranes Using ${ }^{2} \mathrm{H}$ NMR. Biochemistry 1992, 31, 1092-1099.

31. Marcotte, I.; Auger, M., Bicelles as Model Membranes for Solid- and Solution-State NMR Studies of Membrane Peptides and Proteins. Concepts Magn. Reson. Part A 2005, 24A, 17-37.

32. Demco, D. E.; Johansson, A.; Tegenfeldt, J., Proton Spin Diffusion for Spatial Heterogeneity and Morphology Investigations of Polymers. Solid State Nucl. Magn. Reson. 1995, 13-38.

33. Rossini, A. J.; Zagdoun, A.; Hegner, F.; Schwarzwälder, M.; Gajan, D.; Copéret, C.; Lesage, A.; Emsley, L., Dynamic Nuclear Polarization NMR Spectroscopy of Microcrystalline Solids. J. Am. Chem. Soc. 2012, 134, 16899-16908.

34. Rossini, A. J.; Widdifield, C. M.; Zagdoun, A.; Lelli, M.; Schwarzwälder, M.; Copéret, C.; Lesage, A.; Emsley, L., Dynamic Nuclear Polarization Enhanced NMR Spectroscopy for Pharmaceutical Formulations. $J$. Am. Chem. Soc. 2014, 136, 2324-2334.

35. Schlagnitweit, J.; Tang, M.; Baias, M.; Richardson, S.; Schantz, S.; Emsley, L., Nanostructure of Materials Determined by Relayed Paramagnetic Relaxation Enhancement. J. Am. Chem. Soc. 2015, 137, 12482-12485

36. Schlagnitweit, J.; Tang, M.; Baias, M.; Richardson, S.; Schantz, S.; Emsley, L., A Solid-State Nmr Method to Determine Domain Sizes in MultiComponent Polymer Formulations. J. Magn. Reson. 2015, 261, 43-48.

37. Pinon, A. C., et al., Measuring Nano to Micro Structures from Relayed DNP NMR. The Journal of Physical Chemistry C 2017, 121, 15993-16005.

38. van der Wel, P. C. A.; Hu, K.-N.; Lewandowski, J.; Griffin, R. G., Dynamic Nuclear Polarizationof Amyloidogenic Peptide Nanocrystals: GNNQQNY, a Core Segment of the Yeast Prion Protein Sup35p. J. Am. Chem. Soc. 2006, 128, 10810-10846.

39. Jantschke, A.; Koers, E.; Mance, D.; Weingarth, M.; Brunner, E.; Baldus, M., Insight into the Supramolecular Architecture of Intact Diatom Biosilica from DNP-Supported Solid-State NMR Spectroscopy. Angew. Chem. Int. Ed. 2015, 54, 15069-15073.

40. Harris, R. K.; Becker, E. D.; Cabral De Menezes, S. M.; Granger, P.; Hofman, R. E.; Zilm, K. W., Further Conventions for NMR Shielding and Chemical Shifts IUPAC Recommendations 2008. Solid State Nucl. Magn. Reson. 2008, 33, 41-56.

41. Eichele, K., WSolids1 ver. 1.20.21, Universität Tübingen 2013.

42. Pines, A.; Gibby, M. G.; Waugh, J. S., ProtonEnhanced Nuclear Induction Spectroscopy. A Method for High Resolution NMR of Dilute Spins in Solids. $J$. Chem. Phys. 1972, 56, 1776-1777.

43. Lowe, I. J., Free Induction Decays of Rotating Solids. Phys. Rev. Lett. 1959, 2, 285-287.

44. Andrew, R. E.; Bradbury, A.; Eades, R. G., Removal of Dipolar Broadening of Nuclear Magnetic Resonance Spectra of Solids by Specimen Rotation. Nature 1959, 1802-1803. 
45. Nali, B. T.; Rothwell, W. P.; Waugh, J. S.; Rupprecht, A., Structural Studies of a-Form Sodium Deoxyribonucleic Acid: Phosphorus-31 Nuclear Magnetic Resonance of Oriented Fibers. Biochemistry 1981, 20, 1881-1887.

46. Song, Z.; Antzutkin, O. N.; Lee, K. L.; Shekar, S. C.; Rupprecht, A.; Levitt, M. H., Conformational Transitions of the Phosphodiester Backbone in Native DNA: Two-Dimensional Magic-Angle-Spinning 31PNMR of DNA Fibers. Biophys. J. 1997, 73, 1539-1552.

47. Benda, L.; Schneider, B.; Sychrovsky, V., Calculating the Response of NMR Shielding Tensor Sigma $\left({ }^{31} \mathrm{P}\right)$ and ${ }^{2} \mathrm{~J}\left({ }^{31} \mathrm{P},{ }^{13} \mathrm{C}\right)$ Coupling Constants in Nucleic Acid Phosphate to Coordination of the $\mathrm{Mg}^{2+}$ Cation. $J$. Phys. Chem. A 2011, 115, 2385-2295.

48. Benda, L.; Sochorova Vokacova, Z.; Straka, M.; Sychrovsky, V., Correlating the ${ }^{31} \mathrm{P}$ NMR Chemical Shielding Tensor and the ${ }^{2} \mathrm{~J}(\mathrm{P}, \mathrm{C})$ Spin-Spin Coupling Constants with Torsion Angles Zeta and Alpha in the Backbone of Nucleic Acids. J. Phys. Chem. B 2012, 116, 3823-3833.
49. Suzumara, K.-I.; Yoshinari, K.; Tanaka, Y.; Takagi, Y.; Kasai, Y.; Warashina, M.; Kuwabara, T.; Orita, M.; Taira, K., A Reappraisal, Based on ${ }^{31}$ P NMR, of the Direct Coordination of a Metal Ion with the Phosphoryl Oxygen at the Cleavage Site of a Hammerhead Ribozyme. J. Am. Chem. Soc. 2002, 124, 8230-8236.

50. Osborne, E. M.; Ward, W. L.; Ruehle, M. Z.; DeRose, V. J., The Identity of the Nucleophile Substitution May Influence Metal Interactions with the Cleavage Site of the Minimal Hammerhead Ribozyme. Biochemistry 2009, 48, 10654-64.

51. Zhigaltsev, I. V.; Belliveau, N.; Hafez, I.; Leung, A. K.; Huft, J.; Hansen, C.; Cullis, P. R., Bottomup Design and Synthesis of Limit Size Lipid Nanoparticle Systems with Aqueous and Triglyceride Cores Using Millisecond Microfluidic Mixing. Langmuir 2012, 28, 3633-40. 


\title{
Structure of Lipid Nanoparticles Containing siRNA
}

\author{
or mRNA by Dynamic Nuclear Polarization
}

\section{Enhanced NMR Spectroscopy}

\author{
Jasmine Viger-Gravel ${ }^{1}$, Anna Schantz, ${ }^{2}$, Arthur C. Pinon ${ }^{1}$, Aaron J. Rossini ${ }^{1,3}$, Staffan \\ Schantz $^{4}$, and Lyndon Emsley ${ }^{l^{*}}$
}

\begin{abstract}
${ }^{1}$ Institut des Sciences et Ingénierie Chimiques, Ecole Polytechnique Fédérale de Lausanne (EPFL), CH-1015 Lausanne, Switzerland; ${ }^{2}$ Advanced Drug Delivery, Pharmaceutical Sciences, IMED Biotech Unit, AstraZeneca, Gothenburg, Sweden; ${ }^{3}$ Iowa State University, Department of Chemistry, Ames, IA, USA, ${ }^{4}$ Pharmaceutical Technology \& Development, AstraZeneca, Gothenburg, Sweden.
\end{abstract}




\subsection{Characterisation of the LNPs}

Table S1. LNP empty and LNP/cargoe entities DSL measurements. ${ }^{a}$

\begin{tabular}{|c|c|c|c|c|}
\hline Sample & Encapsulation cargo (\%) & $\begin{array}{l}\text { Z-average } \\
(\mathrm{nm})\end{array}$ & $\begin{array}{l}\text { Polydispersity index } \\
\text { (PDI) }\end{array}$ & $\begin{array}{l}\text { Number } \\
\text { average (nm) }\end{array}$ \\
\hline LNP empty & - & 61 & 0.16 & 36 \\
\hline $\begin{array}{l}\text { LNP } 100 \quad \% \\
\text { phosphorothioated siRNA }\end{array}$ & 99 & 52 & 0.12 & 36 \\
\hline $\begin{array}{lc}\text { LNP } & 50\end{array}$ & 96 & 65 & 0.13 & 43 \\
\hline LNP with siRNA & 95 & 53 & 0.06 & 42 \\
\hline LNP mRNA & 98 & 94 & 0.20 & 53 \\
\hline
\end{tabular}

${ }^{a}$ All sizes are obtained after dialysis in PBS 7.4 and before any steps of centrifugation or $\mathrm{D}_{2} \mathrm{O}-$ washing in order to be comparable.

\subsection{Explanation of how the particles do not aggregate at low temperature.}

We have strong evidence that LNPs do not undergo irreversible aggregation at $100 \mathrm{~K}$. In order to be confident that the LNPs would endure the experimental conditions of the DNP SSNMR measurements, e.g. quenching down to $100 \mathrm{~K}$, a repeated freeze-thaw cycle test was performed. A cryo-protectant, 30 vol\% glycerol (UltraPure Glycerol from Invitrogen by Thermo Fisher Scientific), was added to a sample and the sample was then instantly frozen by immersion in liquid nitrogen, thawed and DLS was performed. This procedure was repeated and showed that the freeze-thaw cycles did not have an effect on the particle size and PDI as monitored by DLS (results not shown here) and there seemed to be no risk of freeze induced aggregation of the LNPs. Hence, the LNPs (with and without cargo) size measured by DLS is the same before and after quenching at $100 \mathrm{~K}$. In addition, the LNPs were stable still after $48 \mathrm{hrs}$ when their size distributions and PDI were remonitored by DLS. These finding are supported by cryo-TEM images which show no sign of the LNPs aggregating before and after quenching. Furthermore, our DNP results support that 
LNPs do not aggregate. If the LNP had aggregated, the numerical spin diffusion simulations would have predicted their domain size to have increased which would not be in agreement with the LNP size measure from DLS measurements, and much lower enhancement for the LNP component would be predicted.

\subsection{Preparation of LNPs and DNP samples}

LNP formulation, $\mathrm{D}_{2} \mathrm{O}$ washing, freeze-thaw stability and $x$ RNA cargo encapsulation. $\mathrm{D}_{2} \mathrm{O}-\mathrm{LNP}$ solutions with concentrations of LNP of about $60 \mathrm{mg} / \mathrm{mL}$ tailor-made for DNP-NMR were prepared as follows. Individual lipid stock solutions of the ionizable cationic lipid Dlin-MC3DMA (synthesiezd in-house at AstraZeneca), helper lipid DSPC (Avanti Polar Lipids), Cholesterol (Sigma-Aldrich) and PEGylated lipid DMPE-PEG 2000 (NOF Corporation), were prepared by dissolution in $100 \%$ ethanol and maintained at $4-8^{\circ} \mathrm{C}$. Prior to LNP preparation the lipids were mixed together at a molar percentage ratio of $50 \%$ cationic lipid, $10 \%$ DSPC, $37.5 \%$ cholesterol 1.5\% DMPE-PEG 2k. The LNPs were prepared by mixing appropriate volumes of lipid stock solutions in ethanol with an aqueous phase of $100 \mathrm{mM}$ RNAse free citrate buffer of $\mathrm{pH} 3$ (Teknova) at a 1:3 mixing ratio with a mixing rate of $12 \mathrm{ml} / \mathrm{min}$ using a NanoAssemblr (Precision NanoSystems INc). The setup of this microfluidic mixing instrument is described in detail elsewhere. ${ }^{1}$ The encapsulation of xRNA was determined by the Quant-iT RiboGreen RNA Assay Kit (Thermo Fisher Scientific) on an_EnSpire benchtop multimode plate reader (Perkin Elmer). The encapsulation efficiency of all samples was 96-99\%. The LNPs were then dialyzed for 24 hours against 400x sample volume phosphate-buffered saline (GIBCO by life Technoloigies) at

$\mathrm{pH} 7.4$ at $4-8^{\circ} \mathrm{C}$ using Slide-A-Lyzer ${ }^{\mathrm{TM}} \mathrm{G} 2$ dialysis cassettes with a MWCO of $10 \mathrm{~K}$ (Thermo Fisher Scientific). All the samples were then washed multiple times by the usage of Amicon ultracentrifugation filters with a MWCO of 30k in order to exchange the PBS for 99.8 atom\% 
deuterium oxide (Armar Chemicals). The samples were allowed to equilibrate overnight in between the wash cycles. The total lipid content as well as the individual lipid content of the LNPs were analyzed by LC-MS (CAD detection) in order to confirm that the molar ratios of the lipids was unaffected by the washing procedure (performed only for the first couple of batches, results shown in SI Table S2). The siRNA was a 25 nucleotide duplex strand and obtained from Dharmacon. The siRNA had a $0 \%, 50 \%$ or $100 \%$ phosphorothioated backbone. The mRNA used was Erythropoietin (EPO) (858 nucleotides) ARCA capped modified with 5-methylcytidine and pseudouridine (TriLink Biotechnologies). The empty LNPs, i.e. no xRNA load, were prepared using $100 \mathrm{mM}$ citrate buffer as the aqueous phase. All samples were stored at $4-8^{\circ} \mathrm{C}$.

DNP sample preparation. The LNP samples were prepared with pure $\mathrm{D}_{2} \mathrm{O}$ since partial deuteration of the solvent is usually beneficial for DNP and the components of the LNP are fully protonated (see above). We estimate the overall proton concentration of the LNP formulations to be similar to the $90 \%$ deuterated water-glycerol solutions that typically provide the highest DNP enhancements. Typically, $50 \mu \mathrm{L}$ of ${ }^{12} \mathrm{C}$-glycerol- $d_{8}$ was transferred to an Eppendorf vial (the actual volume of glycerol is accurately determined by weigh, $\varrho=1.25 \mathrm{~g} / \mathrm{ml}) .70 \%$ by volume of the LNP$\mathrm{D}_{2} \mathrm{O}$ dispersion is then added to the glycerol to obtain a solution of ${ }^{12} \mathrm{C}$-glycerol- $d_{8} / \mathrm{LNP}-\mathrm{D}_{2} \mathrm{O}$ sample $(7 / 3 \mathrm{v} / \mathrm{v})$. The biradical polarizing agent AMUPol ( 0.5 to $2 \mathrm{mg})$ was weighed out into a separate Eppendorf vial. The required volume of $\mathrm{LNP} \mathrm{D}_{2} \mathrm{O}-{ }^{12} \mathrm{C}$-glycerol- $d_{8}$ solution is then transferred into the AMUPol vial to provide a final AMUPol concentration of $10 \mathrm{mM}$. Finally, 20$25 \mu \mathrm{L}$ of ${ }^{12} \mathrm{C}$-glycerol- $d_{8} / \mathrm{LNP}-\mathrm{D}_{2} \mathrm{O}$ sample $(3 / 7 \mathrm{v} / \mathrm{v}) 10 \mathrm{mM}$ AMUPol were transferred in a 3.2 mm o.d. saphirre rotor and capped with a silicon plug and zirconia cap. All LNP samples were stored in a $4{ }^{\circ} \mathrm{C}$ refrigerator in between experiments, and new DNP samples were prepared for each analysis, were the LNP- $\mathrm{D}_{2} \mathrm{O}$ dispersion is shaken manually 15 times (not vortexed). 
Table S2. Composition of LNP components in samples 1, 2, 3 and $4 .^{a}$

\begin{tabular}{|c|c|c|c|c|c|c|}
\hline & $\begin{array}{l}\text { DMPE- } \\
\text { PEG }\end{array}$ & Cholesterol & DSPC & $\begin{array}{l}\text { DLin-MC3 } \\
\text {-DMA }\end{array}$ & sum & cargo \\
\hline "MW (g/mol) & 2888 & 387 & 7777 & 643 & & \\
\hline \multicolumn{7}{|l|}{ empty LNP, 1} \\
\hline $\mathrm{m} / \mathrm{v}(\mathrm{mg} / \mathrm{ml})$ & 4.0 & 15.5 & 9.3 & 38.2 & 67 & \\
\hline$\% \mathrm{w} / \mathrm{w}$ & 6.0 & 23.1 & 13.9 & 57.0 & & \\
\hline \multicolumn{7}{|c|}{ LNP ( $100 \%$ phosphorothioated) siRNA, 2} \\
\hline $\mathrm{mv}(\mathrm{mg} / \mathrm{ml})$ & 5.15 & 18.10 & 8.42 & 31.01 & 62.69 & \\
\hline$\% \mathrm{w} / \mathrm{w}$ & 8.23 & 28.87 & 13.43 & 49.46 & & \\
\hline \multicolumn{7}{|c|}{ LNP (50\% phosphorothioated) siRNA, 3} \\
\hline $\mathrm{mv}(\mathrm{mg} / \mathrm{ml})$ & 3.8 & 15.4 & 8.4 & 31.9 & 59.5 & 2.93 \\
\hline$\% \mathrm{w} / \mathrm{w}$ & 6.3 & 25.8 & 14.2 & 53.6 & & \\
\hline \multicolumn{7}{|c|}{ LNP mRNA, 4} \\
\hline $\mathrm{mv}(\mathrm{mg} / \mathrm{ml})$ & 5.35 & 19.12 & 8.54 & 36.93 & 69.94 & \\
\hline$\% \mathrm{w} / \mathrm{w}$ & 7.65 & 27.34 & 12.21 & 52.80 & & \\
\hline
\end{tabular}

\subsection{DNP Acquisition Parameters}

Data were acquired at École Polytechnique Fédérale de Lausanne (EPFL) using a 263 GHz/400 MHz Avance I or III HD Bruker DNP solid-state NMR spectrometer, respectively, equipped with a $3.2 \mathrm{~mm}$ o.d. Bruker triple resonance low temperature magic angle spinning (LTMAS) probe and the experiments were performed at ca. 90-100 K. The sweep coil of the main magnetic field was 
set for the microwave irradiation occurring at the ${ }^{1} \mathrm{H}$ positive enhancement maximum of the AMUPol biradical. Enhancement factor, $\varepsilon$, is the ratio of the signal intensity with and without microwaves.

${ }^{13} \mathrm{C}$ and ${ }^{31} \mathrm{P}$ DNP Enhanced NMR Spectroscopy. For ${ }^{13} \mathrm{C}$ NMR $\left(v_{\mathrm{L}}\left({ }^{13} \mathrm{C}\right)=100.6 \mathrm{MHz}\right.$ at $\left.9.4 \mathrm{~T}\right)$, and ${ }^{31} \mathrm{P}$ NMR $\left(v_{\mathrm{L}}\left({ }^{13} \mathrm{C}\right)=161.9 \mathrm{MHz}\right.$ at $\left.9.4 \mathrm{~T}\right)$, the acquisition parameters used for a typical CPMAS experiment are: $1-2$ s repetition delay, a ${ }^{1} \mathrm{H} \pi / 2$ pulse length of $2.5 \mu$ s to afford $100 \mathrm{kHz}{ }^{1} \mathrm{H}$ decoupling using the SPINAL-64 method, a contact time of 9 and $14 \mathrm{~ms}$ for ${ }^{13} \mathrm{C}$ and ${ }^{31} \mathrm{P}$, respectively, and a spinning frequency of 8,10 or $12.5 \mathrm{kHz}$.

\subsection{DNP enhanced NMR Spectra}

${ }^{l} H$ DNP enhanced NMR-Presented in Figure S1 are the MAS ${ }^{1} \mathrm{H}$ solid echo experiments of the three LNP samples. The isotropic chemical shift values observed are a contribution from the cryo protectant in the solvent, $\left({ }^{12} \mathrm{C}\right.$-glycerol- $d_{8}(98 \%$ deuterated $)$ around $\left.\sim 6 \mathrm{ppm}\right)$, an average distribution of the LNP protons (cover the proton frequency range of 2-10 ppm), as well as a contribution from the silicone plug in the rotor $(0 \mathrm{ppm})$. To verify the stability of LNPs, the spectra where acquired at multiple time intervals. The enhancements are constant with time with an average value around 110 . 
(a)
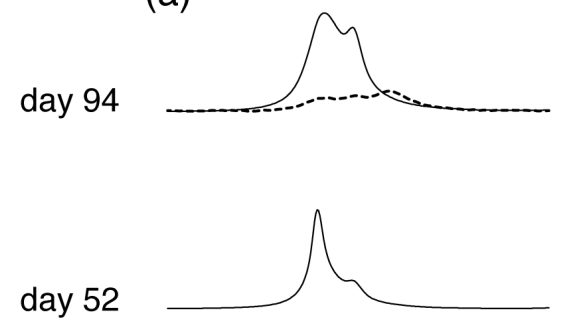

day 32

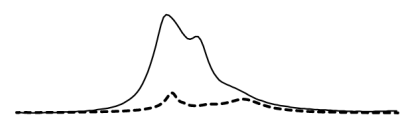

day 7

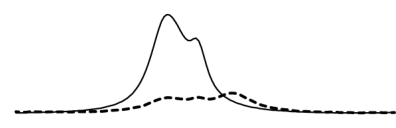

day 1

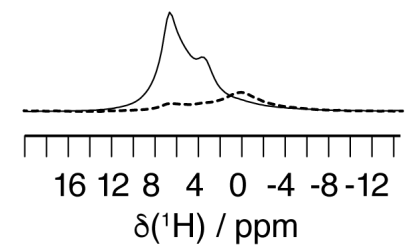

(b)
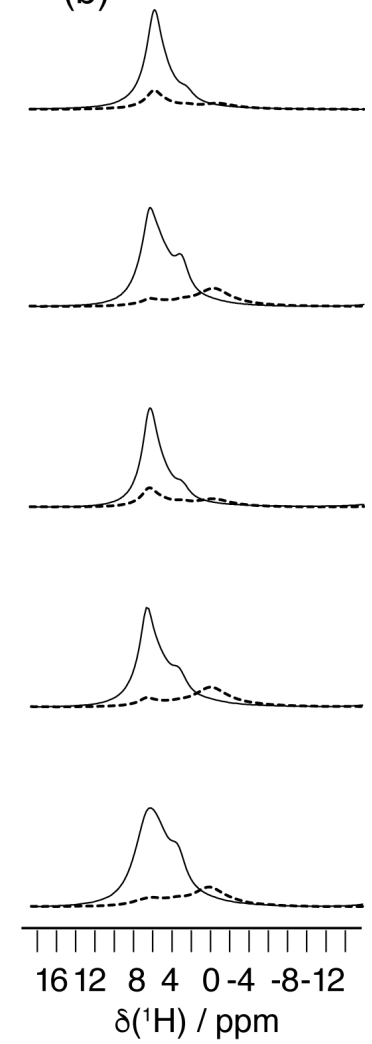

Figure S1. Experimental ${ }^{1} \mathrm{H}$ DNP Hahn- echo MAS spectra acquired after having been stored in the fridge for different days (expressed to the left) with $\mu$ waves on (black trace) and off (dashed trace) for compound (a) 1, (b) 2 spinning at a rate between 8 or $10 \mathrm{kHz}$. The silicone plug is observed on the $\mu$ waves off spectra a 0 ppm. 


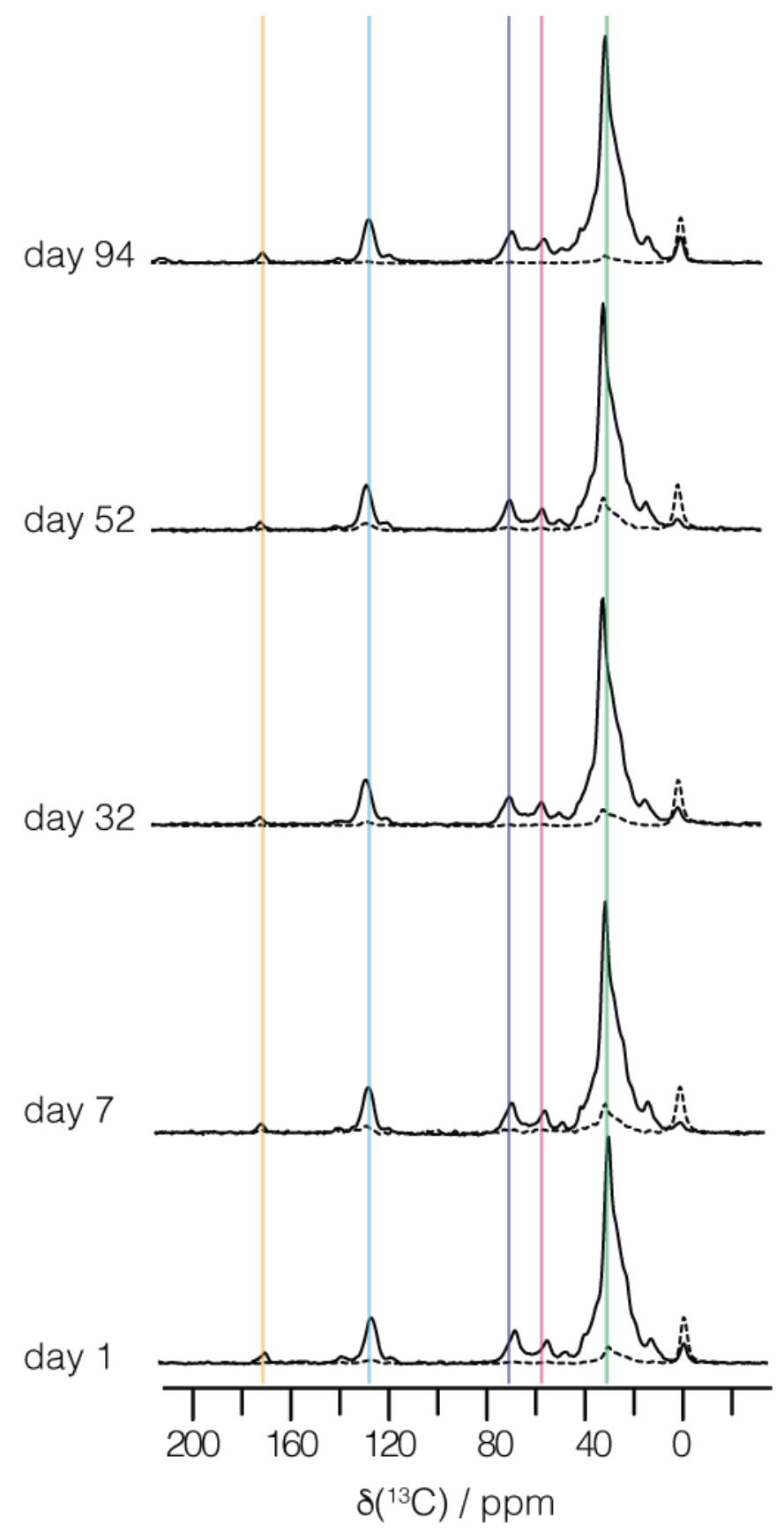

Figure S2. Experimental ${ }^{13} \mathrm{C}$ DNP enhanced CPMAS spectra acquired with a $400 \mathrm{MHz} / 263 \mathrm{GHz}$ DNP system for 1 (LNP empty) after having been stored in the fridge for different time periods (left) with microwave irradiation on (black trace) and off (dashed trace), spinning at a rate between $8 \mathrm{kHz}$ and $12.5 \mathrm{kHz}$, at $100 \mathrm{~K}$, and a recycle delay of $1 \mathrm{~s}$. The silicone plug is observed on the $\mu$ waves on and off spectra a $0 \mathrm{ppm}$. The components of the LNP are resolved in the ${ }^{13} \mathrm{C}$ spectra, they are color coded with respect to the legend described in Figure 1 of the main text and a vertical lines show that the chemical shifts are constant over time. 
Table S3. ${ }^{I} \mathrm{H}$ enhancement values over time for all LNP systems acquired with a recycle delay of $1 s .^{a}$

\begin{tabular}{lll}
\hline Days/System & Empty LNP & LNP with siRNA \\
\hline 1 & $79(2)$ & $106(1)$ \\
7 & $117(3)$ & $86(1)$ \\
32 & - & $176(1)$ \\
52 & $117(3)$ & $58(1)$ \\
94 & $66(2)$ & $135(1)$
\end{tabular}

${ }^{a}$ Error bounds are in parentheses and
$\varepsilon \sqrt{S / N_{o n}{ }^{2}+S / N_{o f f}^{2}}$, where $\mathrm{S} / \mathrm{N}_{\text {on or off }}$ is the signal to noise ratio for microwaves on and off. Table $S 4 .{ }^{13} \mathrm{C}$ enhancement values over time for empty LNP acquired with a recycle delay of $1 \mathrm{~s} .{ }^{a}$

\begin{tabular}{lllll}
\hline Days/resonance & $30 \mathrm{ppm}$ & $56-69 \mathrm{ppm}$ & $127 \mathrm{ppm}$ & $170 \mathrm{ppm}$ \\
\hline 1 & $24(1)$ & $59(3)$ & $28(1)$ & $28(3)$ \\
7 & $40(1)$ & $72(3)$ & $46(2)$ & $130(13)$ \\
32 & $44(1)$ & $91(7)$ & $42(2)$ & $100(25)$ \\
52 & $43(1)$ & $77(4)$ & $41(2)$ & $70(12)$ \\
94 & $55(2)$ & $80(3)$ & $59(2)$ & $73(7)$ \\
\hline
\end{tabular}

${ }^{a}$ See footnote for Table S4.

Table S5. ${ }^{13} \mathrm{C}$ enhancement values over time for LNP with 50\% phosphorothionated siRNA acquired with a recycle delay of $1 \mathrm{~s}{ }^{a}$

\begin{tabular}{lllll}
\hline Days/resonance & $30 \mathrm{ppm}$ & $56-69 \mathrm{ppm}$ & $127 \mathrm{ppm}$ & $170 \mathrm{ppm}$ \\
\hline 1 & $33(1)$ & $149(7)$ & $38(2)$ & $19(3)$ \\
7 & $31(1)$ & $102(5)$ & $43(2)$ & \\
32 & $66(2)$ & $24(1)$ & $26(1)$ & $15(3)$ \\
52 & $21(1)$ & $41(2)$ & $34(1)$ & $32(6)$ \\
94 & $34(1)$ & $63(3)$ & $50(2)$ & \\
\hline
\end{tabular}

${ }^{a}$ See footnote for Table S4. 
Table S6. ${ }^{31} P$ enhancement values over time for all LNP systems acquired with a recycle delay of $0.2 s .^{a}$

\begin{tabular}{llll}
\hline Days/System & $\begin{array}{l}\text { Empty LNP } \\
\text { lipid }\end{array}$ & $\begin{array}{l}\text { LNP with 50 \% phosphorothioated siRNA } \\
\text { lipid }\end{array}$ & siRNA \\
\hline 1 & $56(11)$ & $11(2)$ & $29(15)$ \\
32 & $48(13)$ & $22(2)$ & $30(6)$ \\
\hline
\end{tabular}

${ }^{a}$ See footnote for Table S4.

An external reference, $98 \%$ enriched ${ }^{13} \mathrm{C}=\mathrm{O}$-acetate, was added to compound 2 to verify the enhancement of the radical solution. An external reference is necessary in order to measure a ${ }^{13} \mathrm{C}$ resonance which corresponds to the radical solution because the glycerol used is depleted as well as deuterated $\left({ }^{12} \mathrm{C}\right.$-glycerol- $\left.98 \% d_{8}\right)$ in order to observe the carbon resonances of the LNP components in the 40-80 ppm region. The proton spectrum is not resolved enough to distinguish between the LNP components and the radical solution only. Figure S3 (b) shows the spectrum of $\mathbf{2}$ with the addition of the external reference, and in (b) the spectrum of $\mathbf{2}$ acquired four months later without the addition of acetate. The spectrum was acquired later in order to verify that the addition of an external reference did not affect the LNP spectrum. The spectra for 2 acquired with and without acetate have constant chemical shifts which confirms that the addition of acetate does not affect the integrity of the LNP. The enhancement value for the components of the LNP were low and equal to 11(6) with and without acetate. Furthermore, the enhancement measured on acetate is equal to 98 for sample 2 containing acetate. The higher enhancement of acetate compared to the LNP components shows that it is in proximity to the radical and hence it is located outside the LNPs domain. Furthermore, the value of the acetate in the radical solution is in agreement with the enhancements measured in the proton NMR spectra for the radical solution. 


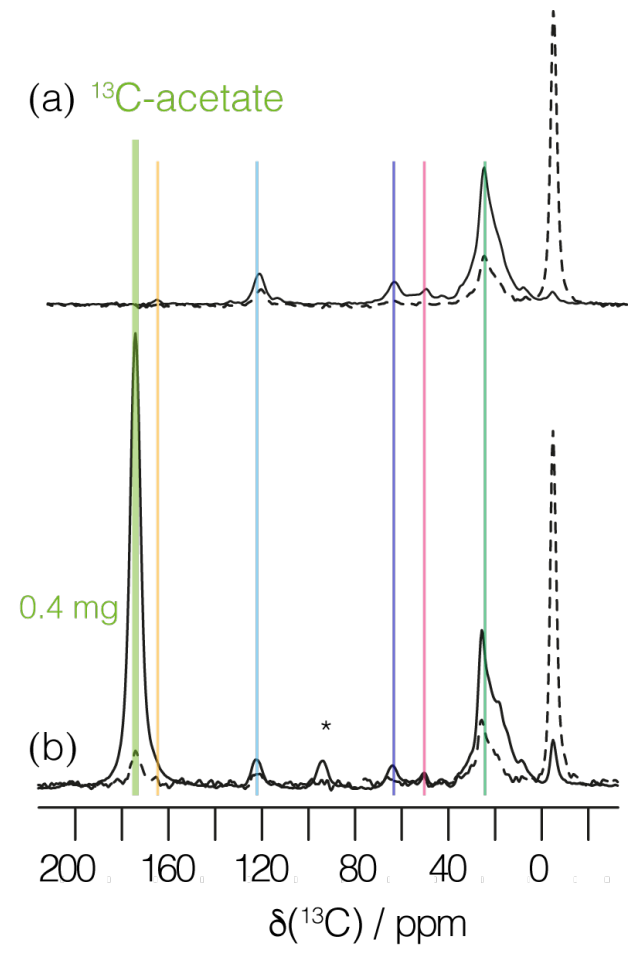

Figure S3. Experimental $\mu$ waves on (black trace) and $\mu$ waves off (dashed line) ${ }^{13} \mathrm{C} \mathrm{CPMAS} \mathrm{DNP}$ spectra acquired at $8 \mathrm{kHz}$ spinning speed for (a) compound 2, and (b) 2 combined with an external reference, ${ }^{13} \mathrm{C}=\mathrm{O}$ labelled-acetate. Resonances follow previous assignment and legend from Figure 2 of main text. 


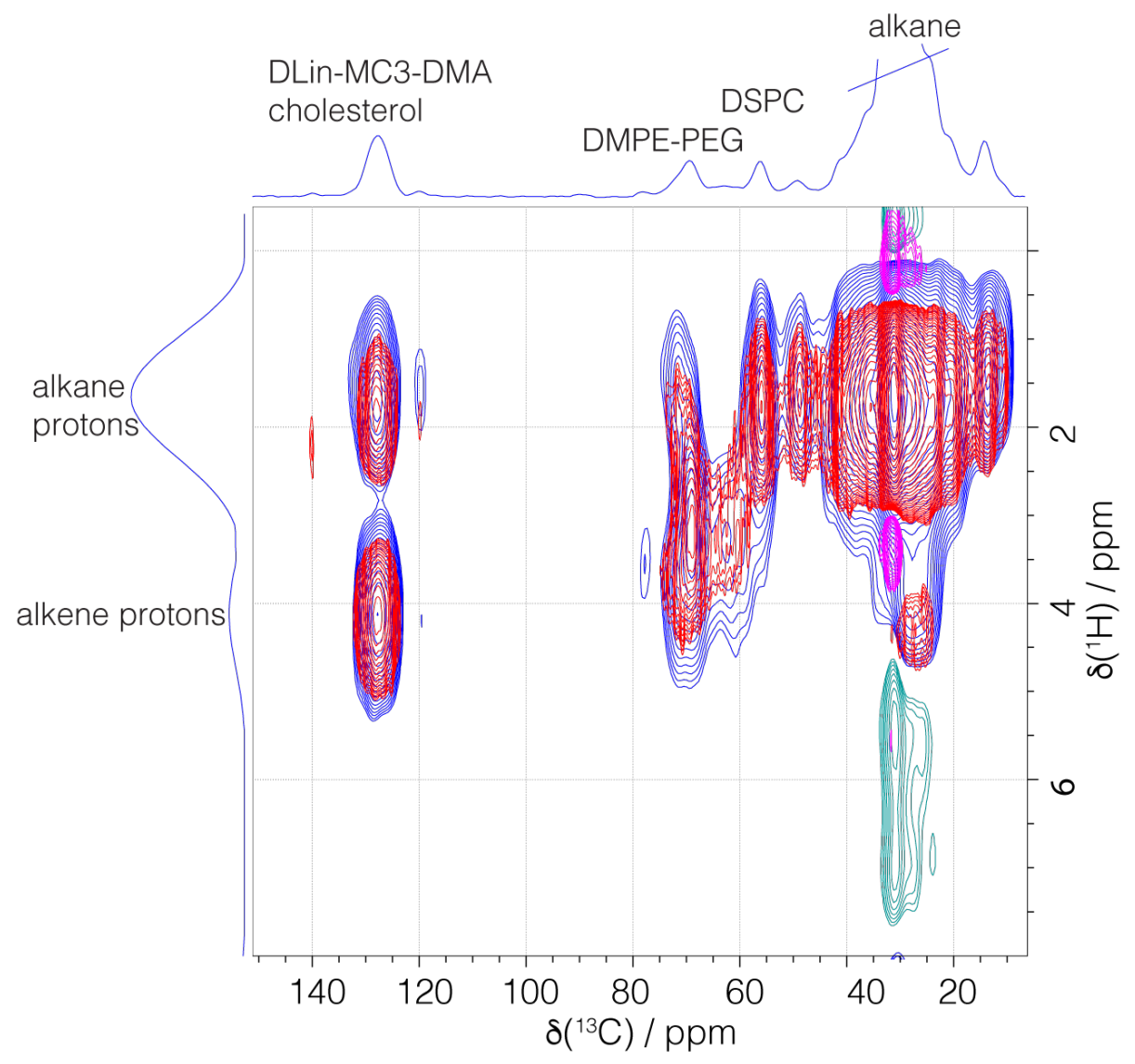

Figure S4. Experimental ${ }^{13} \mathrm{C}$ DNP enhanced ${ }^{1} \mathrm{H}-{ }^{13} \mathrm{C}$ HETCOR spectra acquired for sample 2 (blue) and 4 (red) (MAS $10 \mathrm{kHz}$ ). In the indirect dimension, no chemical shift of the cargo is observed (no cross peak in the proton 7-8 ppm region). The two sample ( 2 and $\mathbf{4}$ ) have the same resonances which corresponds to cross peaks of the LNP components. 

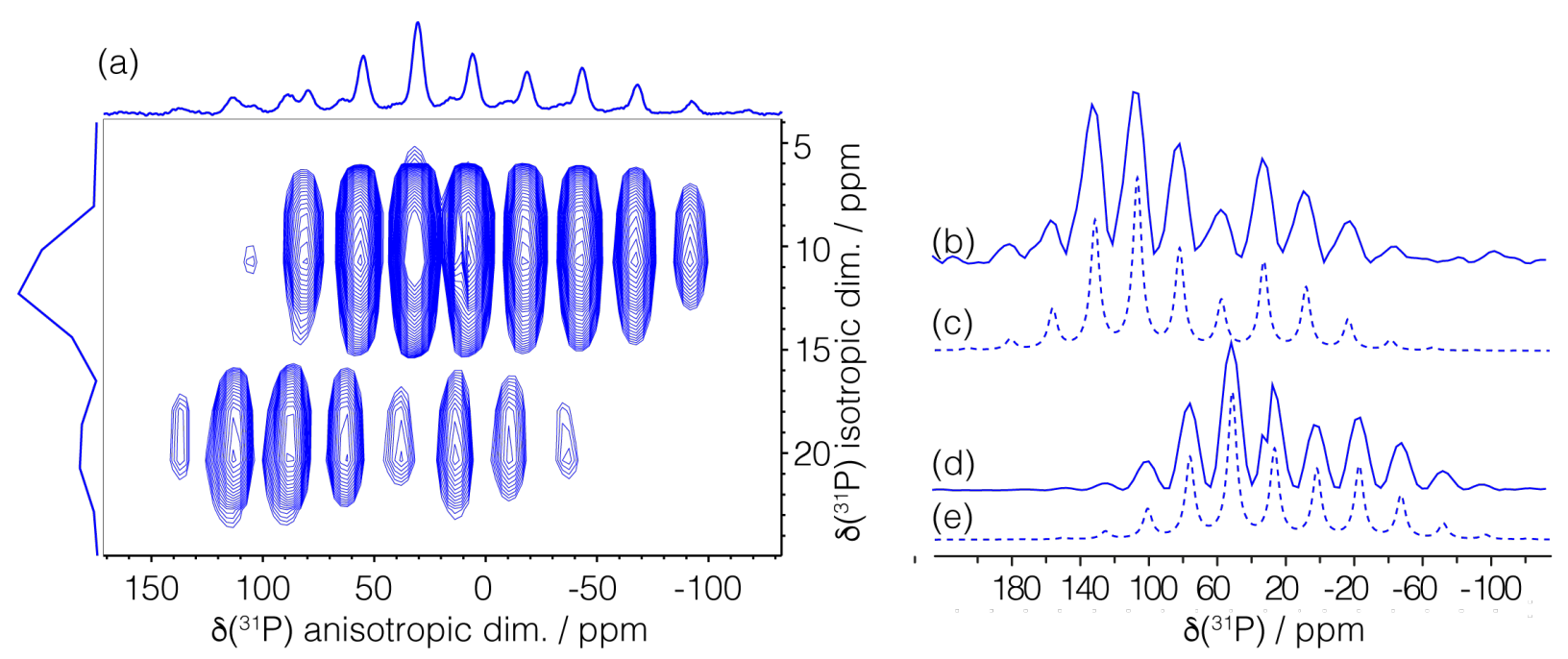

Figure S5. (a) Experimental ${ }^{31} \mathrm{P}_{-}{ }^{31} \mathrm{P}$ CPMAT DNP enhanced solid-state NMR spectrum for compound 3 (blue) acquired at $9.4 \mathrm{~T}$ with a MAS rates of $4 \mathrm{kHz}$. The experimental slices of the CPMAT (right) are extracted and shown in (b-d, blue line) for compound 3. Their simulated spectra are shown represented by dashed lines (c- e, respectively). The lipids (DMPE-PEG and DSPC) and siRNA show distinct/different anisotropy.

\section{Diffusion Model Results 2.1.Build up curves}

All build-up curves where measured with a saturation recovery experiment and fit with a stretched exponential equation which is described below:

$$
S(\tau)=A\left[1-\exp \left(-\left(\frac{\tau}{T_{B}}\right)^{\beta}\right)\right]
$$

where $\mathrm{S}(\tau)$ is the intensity of the signal at the polarization time $\tau, A$ represents the equilibrium non-normalized signal, and beta is the stretching parameter. $T_{\mathrm{B}}$ is the build-up time of the sample at cold temperature with or without microwaves. The $\varepsilon\left({ }^{13} \mathrm{C}\right)$ of the ester functional group of the lipids can only be measured at long polarization delays, as the $\mu$ waves off spectrum for the quaternary carbon is not observable at short delays. 
Table S7. Summary of ${ }^{13}$ C relaxation measurements for empty LNPs for each component measured using $10 \mathrm{mM}$ AMUPol and ${ }^{12} \mathrm{C}$-glycerol-d $d_{8} / L N P(30 / 70 \mathrm{v} / \mathrm{v})$ solution. ${ }^{a}$

\begin{tabular}{llllll} 
resonance $(\mathrm{ppm})$ & $170(3)$ & $127(5)$ & $69(5)$ & $56(7)$ & $30(8)$ \\
\hline$\mu$ waves on & & & & & \\
\hline $\mathrm{A}_{\text {on }}(\mathrm{au})$ & $1.700(0.025)$ & $0.994(0.009)$ & $0.991(0.004)$ & $1.003(0.004)$ & $0.999(0.002)$ \\
$T_{\mathrm{B}}(\mathrm{s})$ & $0.761(0.054)$ & $0.909(0.027)$ & $0.899(0.0157)$ & $0.921(0.013)$ & $0.879(0.006)$ \\
$\beta$ & $0.950(0.104)$ & $0.944(0.041)$ & $0.941(0.024)$ & $0.944(0.019)$ & $0.995(0.009)$ \\
\hline$\mu$ waves off & & & & & \\
\hline $\mathrm{A}_{\text {off }}(\mathrm{au})$ & - & $0.991(0.863)$ & $0.954(0.053)$ & $0.962(0.047)$ & $1.006(0.007)$ \\
$T_{\mathrm{B}}(\mathrm{s})$ & - & $1.587(0.439)$ & $1.224(0.174)$ & $1.425(0.212)$ & $0.943(0.026)$ \\
$\beta$ & - & $0.813(0.222)$ & $1.107(0.235)$ & $1.083(0.227)$ & $0.895(0.034)$ \\
\hline $\bar{\varepsilon}$ & - & $37(2)$ & $55(5)$ & $48(5)$ & $39(1)$
\end{tabular}

\footnotetext{
${ }^{a}$ Error bounds are given in parentheses, and average enhancement value at long polarization time. Errors in the isotropic chemical shifts are the width at half height of the resonance.
}

Table S8. Summary of ${ }^{13} \mathrm{C}$ relaxation measurements of $L N P$ with $100 \%$ phosphorothioated siRNA for each component measured using $10 \mathrm{mM}$ AMUPol and ${ }^{12}$ C-glycerol-d $/$ LNP (30/70 v/v) solution. ${ }^{a}$

\begin{tabular}{llllll} 
resonance $(\mathrm{ppm})$ & $171(3)$ & $127(5)$ & $69(5)$ & $56(8)$ & $29(9)$ \\
\hline$\mu$ waves on & & & & & \\
\hline $\mathrm{A}_{\text {on }}(\mathrm{au})$ & $0.999(0.049)$ & $1.001(0.008)$ & $1.012(0.023)$ & $1.001(0.018)$ & $1.001(0.008)$ \\
$T_{\mathrm{B}}(\mathrm{s})$ & $1.36(0.135)$ & $1.444(0.039)$ & $1.62(0.126)$ & $1.80(0.102)$ & $1.52(0.031)$ \\
$\beta$ & $1.099(0.221)$ & $1.046(0.041)$ & $0.875(0.078)$ & $1.002(0.072)$ & $1.00(0.028)$ \\
\hline$\mu$ waves off & & & & & \\
\hline $\mathrm{A}_{\text {off }}(\mathrm{au})$ & - & $0.928(0.418)$ & $0.894(0.042)$ & $1.083(0.423)$ & $0.967(0.013)$ \\
$T_{\mathrm{B}}(\mathrm{s})$ & - & $1.314(0.223)$ & $1.089(0.125)$ & $3.988(4.393)$ & $1.576(0.069)$ \\
$\beta$ & - & $0.803(0.159)$ & $4.083(1.853)$ & $0.750(0.40)$ & $1.007(0.059)$ \\
\hline $\bar{\varepsilon}$ & - & $31(2)$ & $52(6)$ & $58(8)$ & $29(1)$
\end{tabular}

\footnotetext{
${ }^{a}$ see note Table S7.
} 
Table S9. Summary of ${ }^{13} \mathrm{C}$ relaxation measurements on LNP with $50 \%$ phosphorothioated siRNA for each component measured using $10 \mathrm{mM}$ AMUPol and ${ }^{12}$ C-glycerol-d $/$ LNP (30/70 v/v) solution. ${ }^{a}$

\begin{tabular}{llllll} 
resonance $(\mathrm{ppm})$ & $171(3)$ & $127(5)$ & $69(5)$ & $56(8)$ & $29(9)$ \\
\hline$\mu$ waves on & & & & & \\
\hline $\mathrm{A}_{\text {on }}(\mathrm{au})$ & $0.999(0.03)$ & $0.99(0.01)$ & $1.00(0.01)$ & $1.00(0.01)$ & $1.0(0.002)$ \\
$T_{\mathrm{B}}(\mathrm{s})$ & $1.05(0.13)$ & $1.07(0.039)$ & $1.08(0.02)$ & $1.12(0.04)$ & $1.08(0.009)$ \\
$\beta$ & $1.10(0.20)$ & $1.09(0.05)$ & $0.89(0.03)$ & $1.03(0.05)$ & $1.01(0.01)$ \\
\hline$\mu$ waves off & & & & & \\
\hline $\mathrm{A}_{\text {off }}(\mathrm{au})$ & - & $1.00(0.05)$ & $1.00(0.07)$ & $0.89(0.01)$ & $1.0(0.002)$ \\
$T_{\mathrm{B}}(\mathrm{s})$ & - & $1.73(0.20)$ & $1.85(0.41)$ & $0.96(0.04)$ & $1.08(0.02)$ \\
$\beta$ & - & $1.41(0.28)$ & $0.85(0.03)$ & $1.92(0.05)$ & $0.97(0.2)$ \\
\hline $\bar{\varepsilon}$ & - & $23(2)$ & $45(5)$ & $54(7)$ & $30(1)$ \\
\hline
\end{tabular}

${ }^{a}$ see note Table $\mathrm{S} 7$.

Table S10. Summary of ${ }^{13} \mathrm{C}$ relaxation measurements on LNP containing $m R N A$ for each component measured using $10 \mathrm{mM}$ AMUPol and ${ }^{12} \mathrm{C}$-glycerol-d $d_{8} / L N P(30 / 70 \mathrm{v} / \mathrm{v})$ solution. ${ }^{a}$

\begin{tabular}{lllll} 
resonance $(\mathrm{ppm})$ & $127(5)$ & $69(5)$ & $56(8)$ & $29(9)$ \\
\hline$\mu$ waves on & & & & \\
\hline $\mathrm{A}_{\text {on }}(\mathrm{au})$ & $0.98(0.01)$ & $0.99(0.01)$ & $0.99(0.02)$ & $0.99(0.003)$ \\
$T_{\mathrm{B}}(\mathrm{s})$ & $2.22(0.07)$ & $2.14(0.09)$ & $2.59(0.12)$ & $2.24(0.02)$ \\
$\beta$ & $0.98(0.04)$ & $0.96(0.04)$ & $0.91(0.04)$ & $0.98(0.01)$ \\
\hline$\mu$ waves off & & & & \\
\hdashline $\mathrm{A}_{\text {off }}(\mathrm{au})$ & $1.08(0.03)$ & $0.89(0.17)$ & $1.45(0.15)$ & $0.99(0.07)$ \\
$T_{\mathrm{B}}(\mathrm{s})$ & $1.73(0.16)$ & $2.88(1.44)$ & $2.09(0.59)$ & $2.34(0.05)$ \\
$\beta$ & $0.96(0.11)$ & $1.08(0.58)$ & $1.28(0.57)$ & $1.03(0.03)$ \\
\hdashline $\bar{\varepsilon}$ & $28(1)$ & $58(10)$ & $49(9)$ & $44(4)$
\end{tabular}

${ }^{a}$ see note Table S7. 
Table S11. Summary of ${ }^{31} P$ relaxation measurements of sample 1 and 2 for each component measured using $10 \mathrm{mM}$ AMUPol and ${ }^{12} \mathrm{C}$-glycerol-d8/LNP (30/70 v/v) solution. ${ }^{a}$

\begin{tabular}{|c|c|c|c|c|c|c|c|}
\hline $\begin{array}{l}\text { resonance } \\
(\mathrm{ppm})\end{array}$ & $1(10 \mathrm{ppm})$ & $2(10 \mathrm{ppm})$ & $2(67 \mathrm{ppm})$ & $\operatorname{siRNA} / \mathrm{D}_{2} \mathrm{O}$ & $\begin{array}{l}3(10 \\
\text { ppm })\end{array}$ & $\begin{array}{l}3(67 \\
\text { ppm) }\end{array}$ & 4 \\
\hline \multicolumn{8}{|l|}{$\mu$ waves on } \\
\hline $\mathrm{A}_{\mathrm{on}}(\mathrm{au})$ & $\begin{array}{l}1.000 \\
(0.023)\end{array}$ & $\begin{array}{l}0.982 \\
(0.013)\end{array}$ & $\begin{array}{l}0.985 \\
(0.011)\end{array}$ & $\begin{array}{l}1.044 \\
(0.052)\end{array}$ & $0.99(0.01)$ & $1.00(0.01)$ & $1.00(0.01)$ \\
\hline$T_{\mathrm{B}}(\mathrm{s})$ & $\begin{array}{l}0.699 \\
(0.039)\end{array}$ & $\begin{array}{l}1.702 \\
(0.076)\end{array}$ & $\begin{array}{l}1.739 \\
(0.069)\end{array}$ & $\begin{array}{l}1.121 \\
(0.004)\end{array}$ & $1.26(0.02)$ & $1.29(0.02)$ & $2.20(0.03)$ \\
\hline$\beta$ & $\begin{array}{l}1.098 \\
(0.082)\end{array}$ & $\begin{array}{l}1.033 \\
(0.067)\end{array}$ & $\begin{array}{l}1.007 \\
(0.056)\end{array}$ & $\begin{array}{l}1.000 \\
(0.050)\end{array}$ & $1.00(0.02)$ & $1.01(0.02)$ & $0.97(0.02)$ \\
\hline \multicolumn{8}{|l|}{$\mu$ waves off } \\
\hline $\mathrm{A}_{\text {off }}(\mathrm{au})$ & $\begin{array}{l}1.023 \\
(0.028)\end{array}$ & $\begin{array}{l}0.983 \\
(0.019)\end{array}$ & $\begin{array}{l}0.958 \\
(0.0244)\end{array}$ & - & $1.00(0.02)$ & $0.99(0.07)$ & $0.97(0.03)$ \\
\hline$T_{\mathrm{B}}(\mathrm{s})$ & $\begin{array}{l}0.868 \\
(0.056)\end{array}$ & $\begin{array}{l}1.503 \\
(0.099)\end{array}$ & $\begin{array}{l}1.874 \\
(0.154)\end{array}$ & - & $1.23(0.06)$ & $1.43(0.26)$ & $2.63(0.26)$ \\
\hline$\beta$ & $\begin{array}{l}1.065 \\
(0.076)\end{array}$ & $\begin{array}{l}1.159 \\
(0.130)\end{array}$ & $\begin{array}{l}1.108 \\
(0.133)\end{array}$ & - & $1.05(0.05)$ & $1.21(0.28)$ & $0.91(0.09)$ \\
\hline$\varepsilon$ & $27(2)$ & $41(2)$ & $29(1)$ & - & $50(5)$ & $54(4)$ & $44(5)$ \\
\hline
\end{tabular}

\footnotetext{
${ }^{a}$ see note Table S7.
} 


\subsection{Spin Diffusion Numerical Model}

The proton-proton dipolar coupling dynamics such as spin diffusion simulations were performed using the MATLAB software. The classical diffusion model is used and is previously described in detail by Schlagnitweit et al. ${ }^{2}$, Rosssini et al. ${ }^{3}$, or Pinon et al. ${ }^{4}$

If we assumed that polarization dynamics follows the same behavior as thermal transfer, the process may be described by classical diffusion process, the $2^{\text {nd }}$ Fick law:

$$
\frac{\partial P(x, t)}{\partial t}=D(x) \cdot \Delta P(x, t)-\frac{P(x, t)-P_{o}(x)}{T_{1}(x)}[\mathrm{E} .2]
$$

with the following initial and boundary conditions:

$$
\begin{aligned}
& P(x, 0)=0[\text { E. } 3] \\
& \frac{\partial P\left(x_{0}, t\right)}{\partial x}=0[\text { E. } 4]
\end{aligned}
$$

where $x$ is the distance from the center of the LNP in $\mu \mathrm{m}, x_{\mathrm{o}}$ is the position at the extremity of the system, $t$ is the time in seconds $(0<t<\infty), P$ and $P_{0}$ is the spontaneous and local equilibrium polarization, respectively, $D$ is the diffusion rate constant at point $x, T_{1}$ is the spin-lattice relaxation rate at point $x$, and $\Delta \mathrm{P}$ is the Laplacian polarization which is dependent on the symmetry of the system, and here for LNPs it is spherical. Equation [E.2] may be solved using the following approximations, such that the boundary condition presented in equation [E.3] and [E.4] designates no initial polarization within the system as well as absence of polarization at the border of the system, respectively.

For the LNPs, the size distribution measured by DLS measurements is reproduced using a Weibull distribution (see Figure S6 (a)). The spin diffusion behavior is simulated for all size distribution (Figure S6 (b)). Next the sum of the weighted enhancements as a function of polarization delay is the best fit and is shown on the final figures (Figure S6 (c - d)). The same steps are followed for the buildup curves. All experimental parameters used for the modelling are 
detailed Table S12. Modeling the size distribution was done to convince ourselves that using the number average determined by DLS is enough for the following itirative steps used in the numerical spin diffusion simulations for Model I and II.
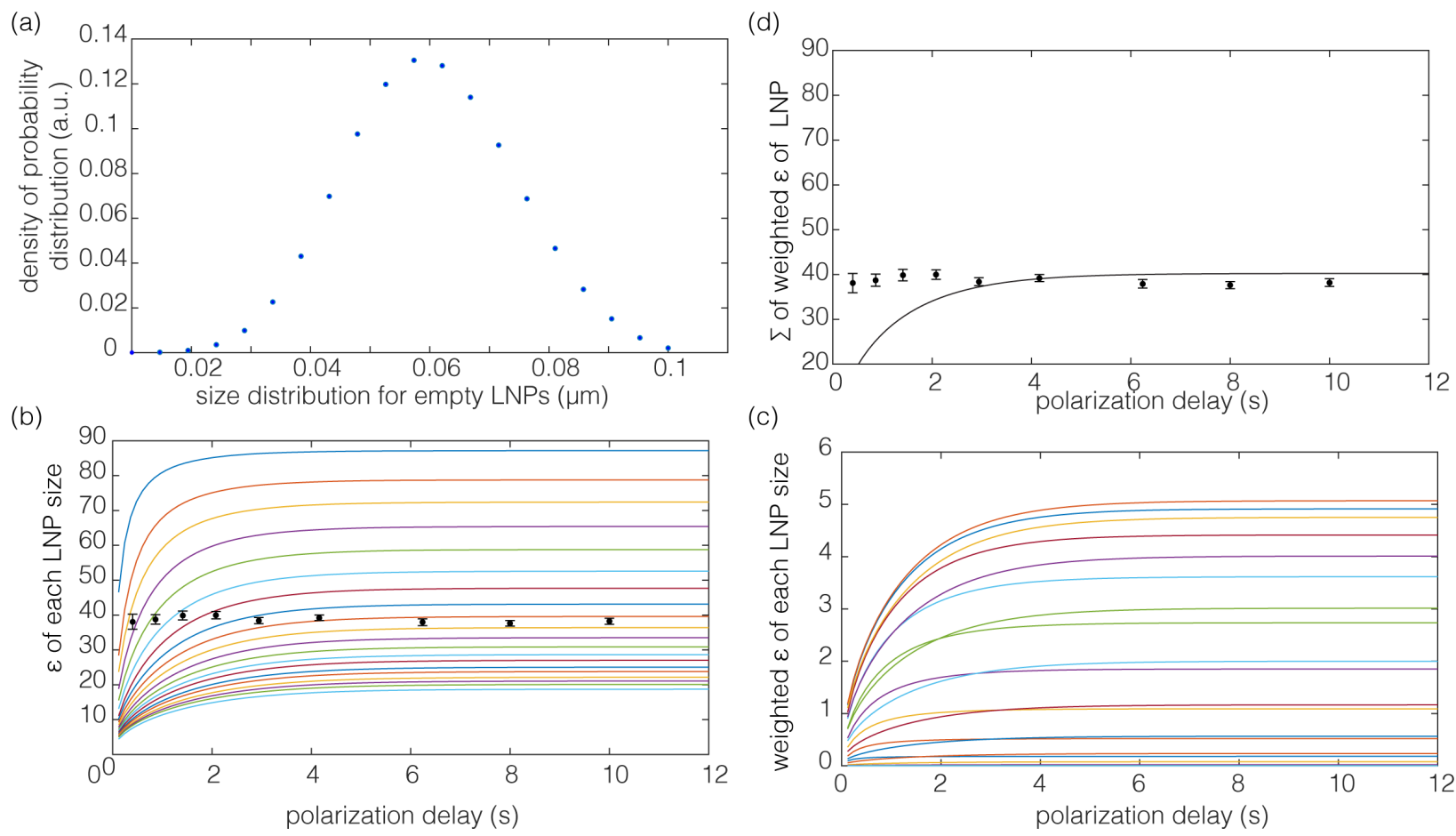

Figure S6. Example of model I proton spin diffusion for LNP empty, alkane peak (30 ppm). (a) Size distribution of LNPs using a Weibull distribution (max, width: 0.055, 4). (b) Enhancement as a function of polarization delay for each size of the LNP distribution, and in (c) is their weighted enhancement. (d) Sum of the weighted enhancement as a function of the polarization delay which gives the best fit. 
Table S12. ${ }^{13} \mathrm{C}$ spin diffusion parameters determined experimentally and used to numerically model the enhancement as a function polarization delay for LNP samples. ${ }^{a}$

\begin{tabular}{|c|c|c|c|c|c|c|c|c|c|c|c|}
\hline sym & $\operatorname{radius}(\mu \mathrm{m})$ & \#steps & $\mathrm{t}_{\max } / \mathrm{s}$ & $\mathrm{t}_{\text {step }} / \mathrm{s}$ & $\begin{array}{l}T_{\text {lout }} \\
\text { (s) }\end{array}$ & $\begin{array}{l}T_{\text {lin }} \\
\text { (s) }\end{array}$ & $\varepsilon_{\text {sol }}$ & $\begin{array}{l}D_{\text {out }} \\
\left(\mu \mathrm{m}^{2} \mathrm{~s}^{-1}\right)\end{array}$ & Pol & $\begin{array}{l}\text { de } \\
\text { pol }\end{array}$ & slope \\
\hline spherical & $0.1+\operatorname{dist}(\mathrm{k})$ & 100 & 12 & 101 & 1.24 & 1.41 & 96 & $1 \cdot 10^{-3}$ & 0.1 & 0.5 & 60000 \\
\hline
\end{tabular}

${ }^{a}$ The LNPs are simulated as spheres, the radius is modeled on the size distribution measured by DLS (see Table S1) using the Weibull distribution which depends on the parameter (max, width); spatial steps are used for the numerical simulation (\#steps); number of points $\left(\mathrm{t}_{\mathrm{max}}\right)$ are calculated for the numerical simulation in a number of steps $\left(t_{\text {steps }}\right)$; the enhancement of the radical solution $\left(\varepsilon_{\text {sol }}\right.$ ), the intrinsic $T_{1}$ (spin-lattice relaxation at $100 \mathrm{~K}$ without radical) of the LNP components $\left(T_{1, \text { in }}\right)$ and the build-up time of the DNP matrix $\left(T_{1, \text { out }}\right)$. The hyperpolarization (pol) and depolarization (dePol) through the radical solution and core are simulated, a hysteresis slope (slope) is used to model the passage between the exterior toward the inside of the domain.

Two ${ }^{1} \mathrm{H}-{ }^{1} \mathrm{H}$ spin diffusion models are used to simulate the structure of LNPs. Model I mimics a homogeneous core model where all components are distributed equally in the domain. We expect the behavior of the enhancement as a function of polarization delay to be the same for the various components of the LNP. For the numerical spin diffusion simulations of model I, only one parameter is optimized $\left(D_{\text {in }}\right)$ iteratively until the minimum value is found. The spin diffusion coefficient inside the LNPs is expected to be different than the value found outside the LNP domain. The numerical spin diffusion model II aims to mimic a lipid layer at the surface of the LNP such as suggests the nanostructured core model. Model II simulates a layer core shell model were the polarization located in the layer and in the core, are integrated separately, and the entire sphere is integrated to provide the value of the sub-core. Model II provides different enhancement values for the various components depending on their spatial location; the $\varepsilon$ values for the components at the surface will be higher than the value for the components located at the core. For the numerical spin diffusion simulations of model II, three parameters are optimized iteratively 
until the minimum values are found (cycles are performed), the spin diffusion coefficient inside $\left(D_{\text {in }}\right)$ and in the layer $\left(D_{\text {lay }}\right)$ of the LNPs as well as the layer length $(L)$. It should be noted that the spin diffusion models systematically fit the experimental build-up curves inversely, i.e. the simulated build-up $\mu$ waves on are fit to the observed $\mu$ waves off data and vice versa. We believe this is a consequence of the large errors of the $\mu$ waves off build-up curves due to low $\mathrm{S} / \mathrm{N}$ and small $T_{\mathrm{B}}$ values that are close for $\mu$ waves on and off.

Table S13. Summary of outputted optimized values (minimum, last cycle) of numerical spin diffusion calculations for model I and II.

\begin{tabular}{|c|c|c|c|c|c|c|c|c|}
\hline & \multicolumn{4}{|c|}{ Model II- Layer model } & \multicolumn{4}{|c|}{ Model I- Homogeneous core } \\
\hline & 1 & 2 & 3 & 4 & 1 & 2 & 3 & 4 \\
\hline $\begin{array}{c}D_{\text {in }} \\
\left(1 \cdot 10^{3} \mu \mathrm{m}^{2} \mathrm{~s}^{-}\right. \\
1)\end{array}$ & $\begin{array}{c}0.12(7.68 \\
8)\end{array}$ & $\begin{array}{c}0.11(4.25 \\
87)\end{array}$ & $\begin{array}{c}0.7(27.664 \\
8)\end{array}$ & $\begin{array}{c}0.2(27.39 \\
16)\end{array}$ & $\begin{array}{c}0.22(14.74 \\
52)\end{array}$ & $\begin{array}{c}0.16(20.74 \\
86)\end{array}$ & $\begin{array}{c}0.23(39.52 \\
97)\end{array}$ & $\begin{array}{c}0.162(20.74 \\
86)\end{array}$ \\
\hline $\begin{array}{c}D_{\text {lay }}\left(1 \cdot 10^{3} \mu\right. \\
\left.\mathrm{m}^{2} \mathrm{~s}^{-1}\right)\end{array}$ & $\begin{array}{c}0.1(7.738 \\
1)\end{array}$ & $\begin{array}{c}0.11(4.66 \\
64)\end{array}$ & $\begin{array}{c}0.12(27.10 \\
86)\end{array}$ & $\begin{array}{c}0.3(26.98 \\
04)\end{array}$ & - & - & & \\
\hline $\begin{array}{l}\text { Layer }\left(1 \cdot 10^{3}\right. \\
\mu \mathrm{m})\end{array}$ & $\begin{array}{c}1.2(7.788 \\
3)\end{array}$ & $\begin{array}{c}5.0(4.669 \\
4)\end{array}$ & $\begin{array}{c}2.4(27.664 \\
8)\end{array}$ & $\begin{array}{c}5.5(28.57 \\
40)\end{array}$ & - & - & & \\
\hline
\end{tabular}



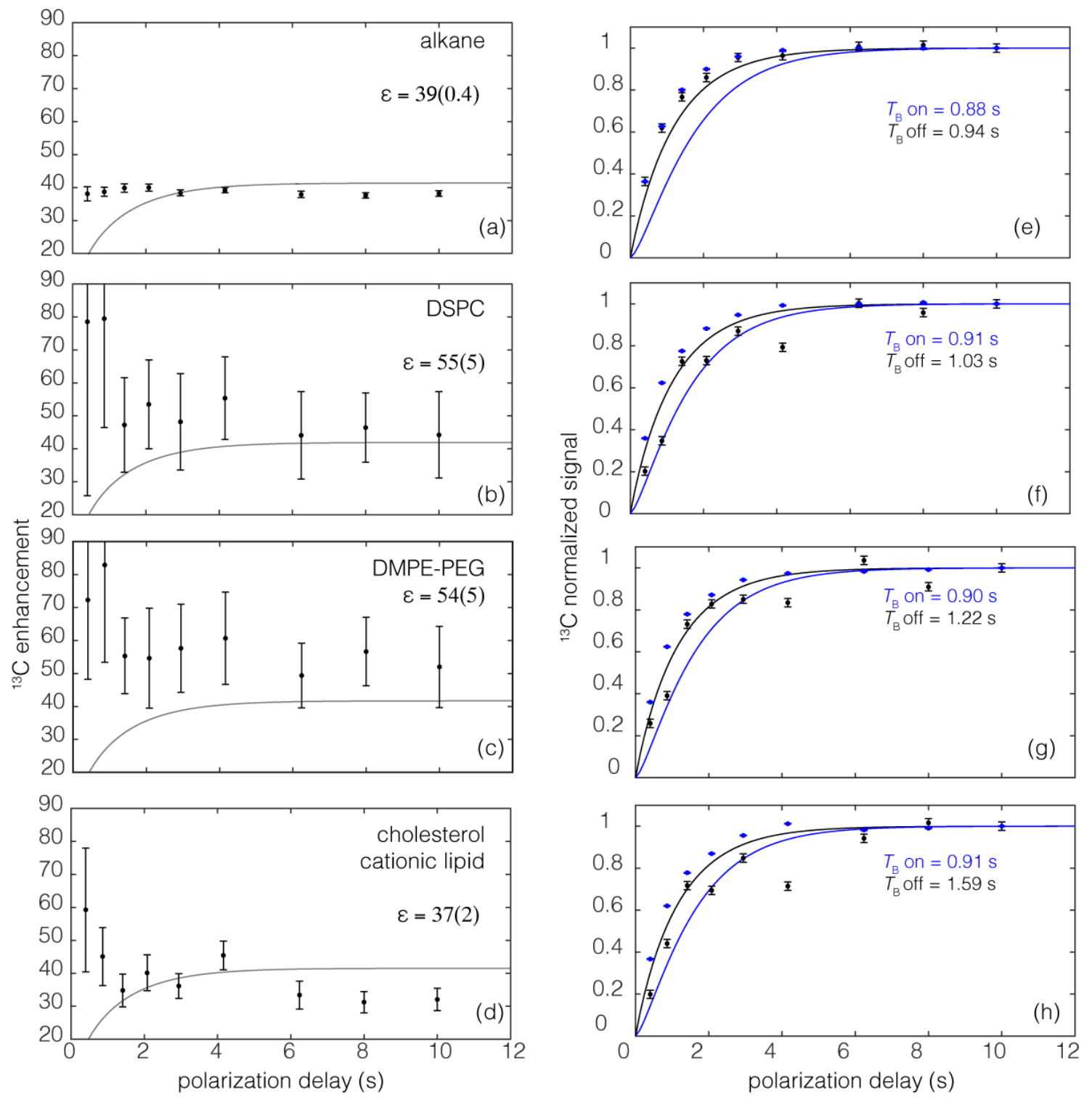

Figure S7. (a) Experimental plots of the enhancement $\left({ }^{13} \mathrm{C}\right)$ values as a function of polarization time (s) for empty LNP chemical shifts at (a) 30 ppm, (b) 57 ppm, (c) 69 ppm (d) 128 ppm. For each resonance, inset to the right are indicated the average enhancement value as well as the assigned component of the resonance. Their respective ${ }^{1} \mathrm{H}-{ }^{1} \mathrm{H}$ spin diffusion modeling are overlaid (grey traces) using spin diffusion model I (homogeneous domain). Simulated build-up curves for microwaves on (black) and off (blue) are overlaid on the experimental data for the resonances at (e) $30 \mathrm{ppm}$, (f) $57 \mathrm{ppm}$, (g) $69 \mathrm{ppm}$ (h) $128 \mathrm{ppm}$ acquired with $\mu$ waves on (blue) and off (black). The $T_{\mathrm{B}}$ on and off values in the inset of the plot are obtained from the analytical fit using the stretched exponential function. 

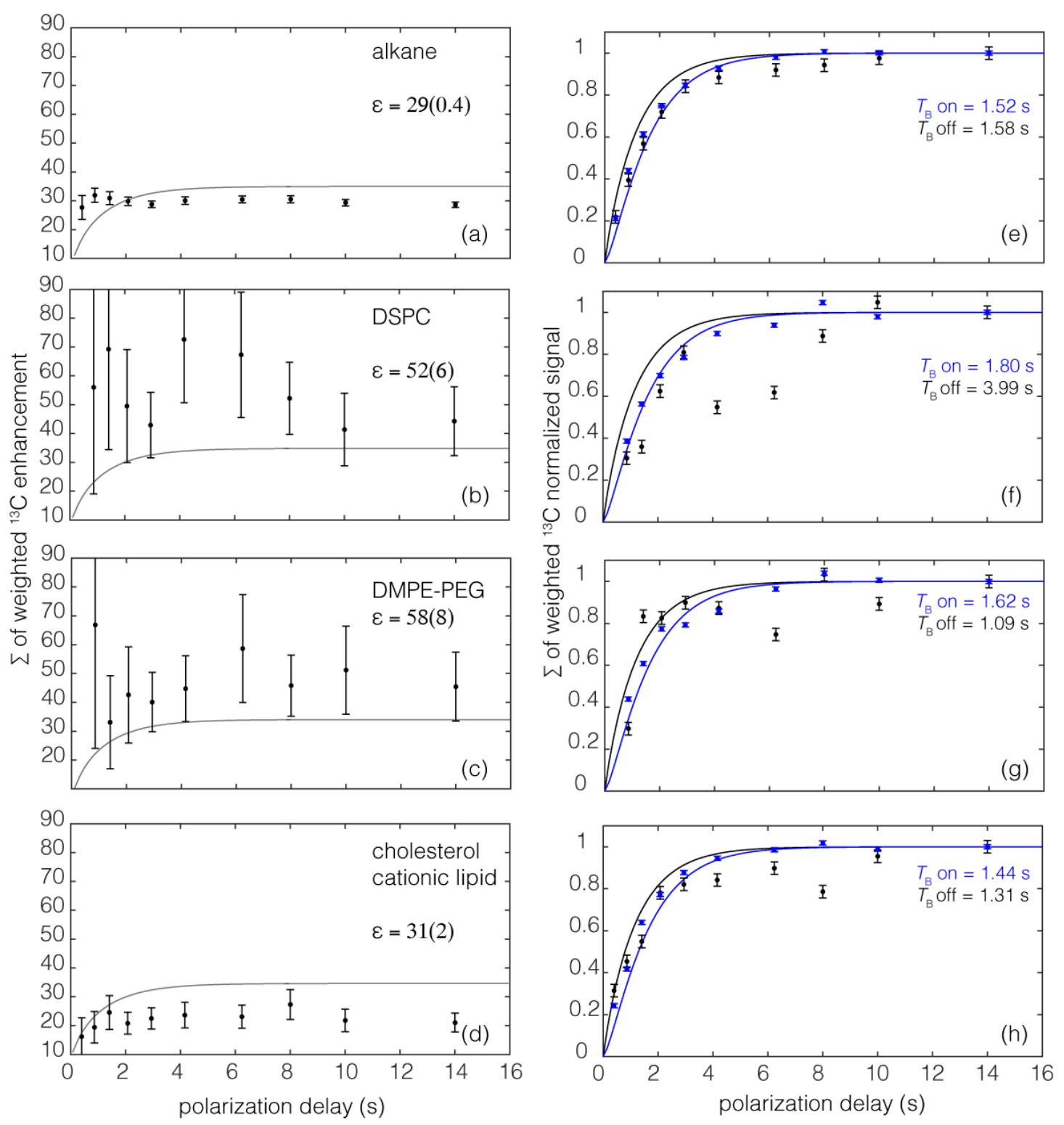

Figure S8. (a) Experimental (circles) plots of the enhancement $\left({ }^{13} \mathrm{C}\right)$ values as a function of polarization time (recycle delay, s) for LNP containing 100\% phosphorothioated siRNA chemical shifts at (a) $30 \mathrm{ppm}$, (c) $56 \mathrm{ppm}$ (e) $69 \mathrm{ppm}$ and (g) $127 \mathrm{ppm}$ as well as their simulation using the spin diffusion model I (gray traces). For each resonance, inset to the right are indicated the average enhancement value as well as the assigned component of the resonance. Their simulated $T_{\mathrm{B}}$ curves are shown for $\mu$ wave (black) and off (blue) are overlaid on the experimental data for the resonances at (e) $30 \mathrm{ppm}$, (f) $57 \mathrm{ppm}$, (g) $69 \mathrm{ppm}$ (h) $128 \mathrm{ppm}$ acquired with $\mu$ wave on (blue) and off (black). Their $T_{\mathrm{B}}$ values are shown on the plots are result from an analytical fit using a stretched exponential function. 

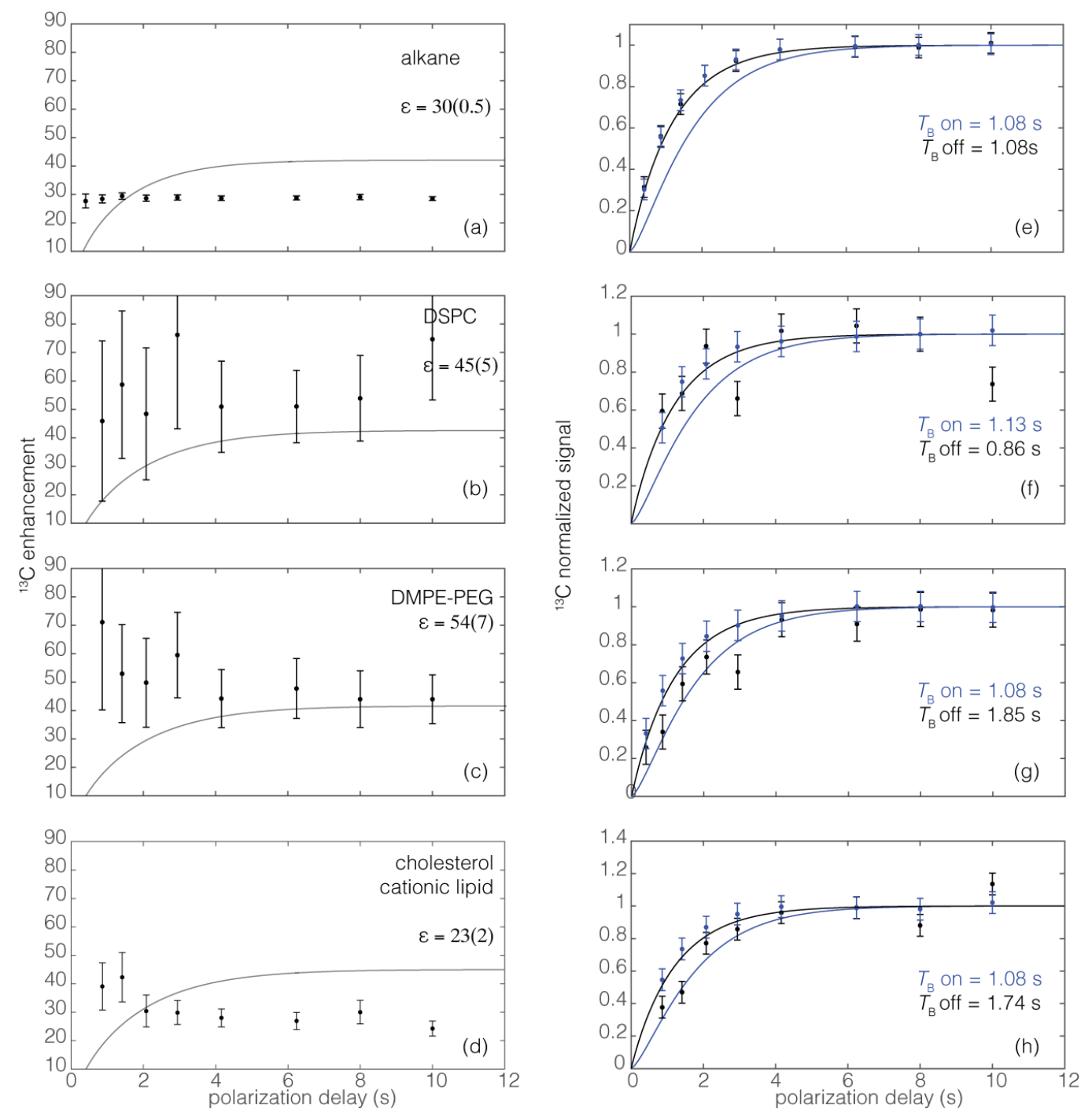

Figure S9. (a) Experimental (circles) plots of the enhancement $\left({ }^{13} \mathrm{C}\right)$ values as a function of polarization time (polarization delay, s) for LNP containing 50\% phosphorothioated siRNA chemical shifts at (a) $30 \mathrm{ppm}$, (c) $56 \mathrm{ppm}$ (e) $69 \mathrm{ppm}$ and (g) $127 \mathrm{ppm}$ as well as their simulation using the spin diffusion model I (grey traces). For each resonance, inset to the right are indicated the average enhancement value as well as the assigned component of the resonance. Their simulated $T_{\mathrm{B}}$ curves are shown for $\mu$ waves on (black) and off (blue) are overlaid on the experimental data for the resonances at (e) $30 \mathrm{ppm}$, (f) $57 \mathrm{ppm}$, (g) $69 \mathrm{ppm}$ (h) $128 \mathrm{ppm}$ acquired with $\mu$ waves on (blue) and off (black). Their analytical $T_{\mathrm{B}}$ values are shown on the plots are result from an analytical fit using a stretched exponential function. 

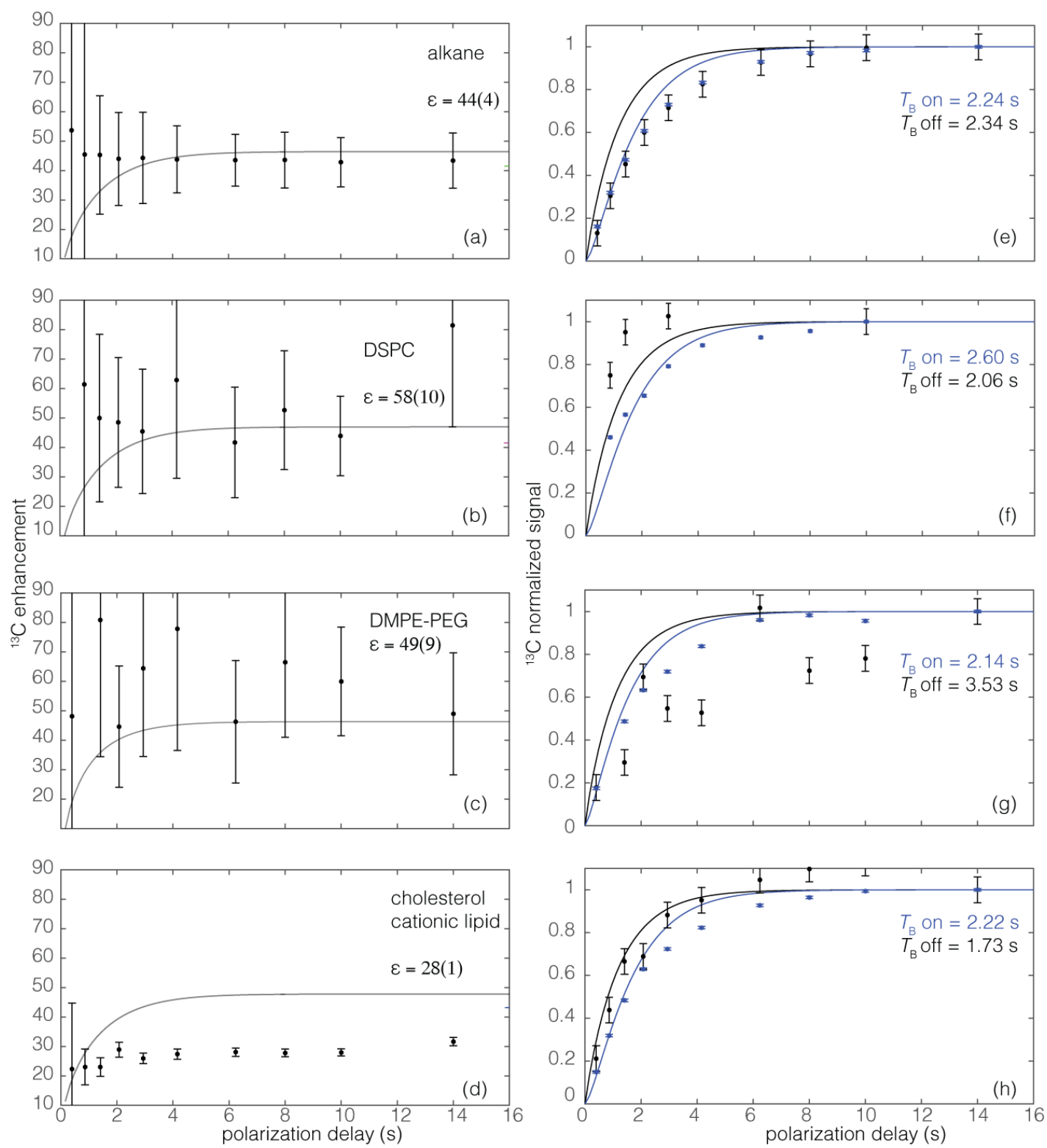

Figure S10. (a) Experimental (circles) plots of the enhancement $\left({ }^{13} \mathrm{C}\right)$ values as a function of polarization time (polarization delay, s) for LNP containing mRNA chemical shifts at (a) $30 \mathrm{ppm}$, (c) $56 \mathrm{ppm}$ (e) $69 \mathrm{ppm}$ and (g) $127 \mathrm{ppm}$ as well as their simulation using the spin diffusion model I (grey traces). For each resonance, inset to the right are indicated the average enhancement value as well as the assigned component of the resonance. Their simulated $T_{\mathrm{B}}$ curves are shown for $\mu$ waves (black) and off (blue) are overlaid on the experimental data for the resonances at (e) 30 ppm, (f) 57 ppm, (g) 69 ppm (h) 128 ppm acquired with $\mu$ waves on (blue) and off (black). Their analytical $T_{\mathrm{B}}$ values are shown on the plots are result from an analytical fit using a stretched exponential function. 

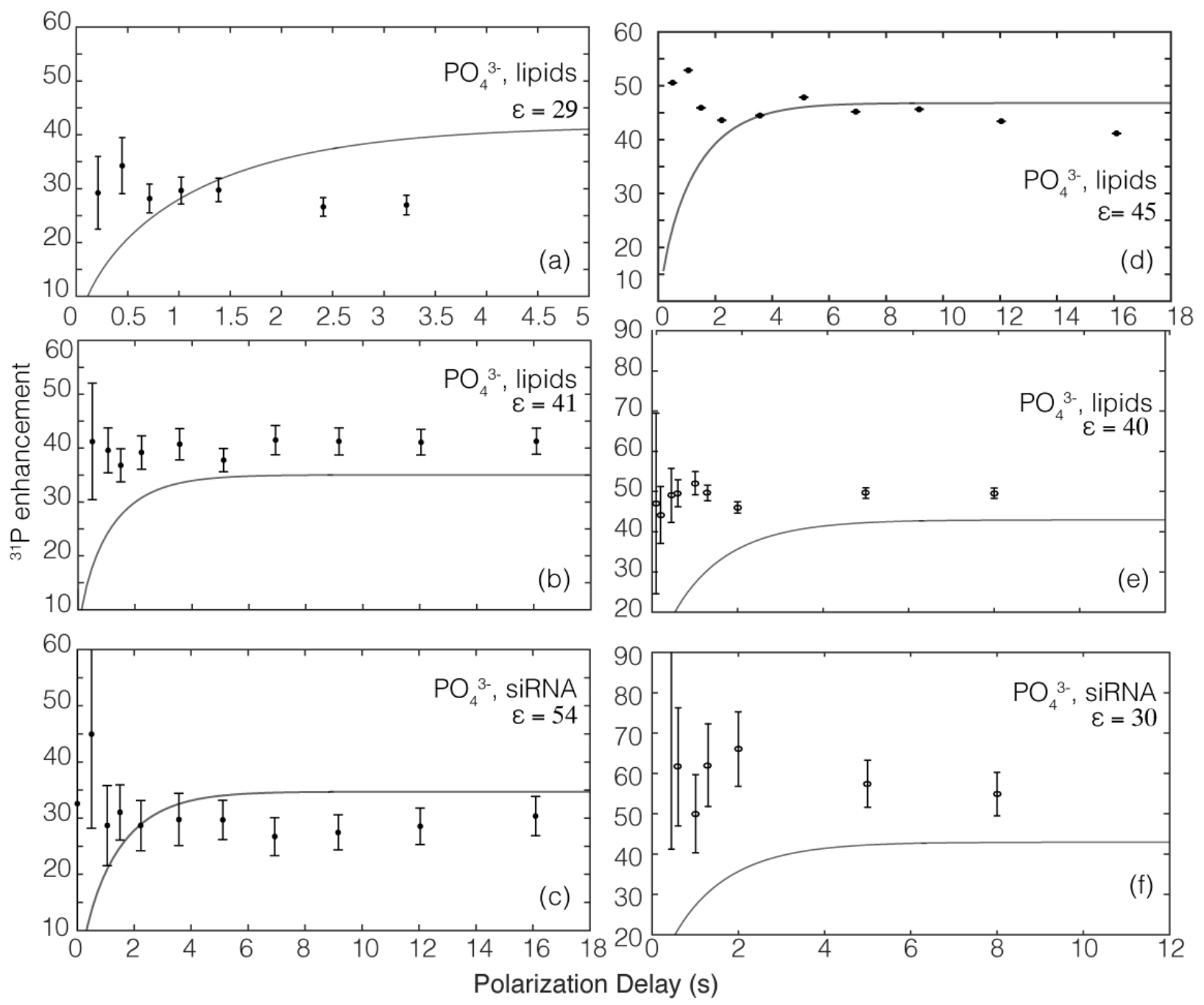

Figure S11. Experimental (black circles) plots of the enhancement $\left({ }^{31} \mathrm{P}\right)$ values as a function of polarization delay for empty LNP (a) and in the presence of the cargo (b) $50 \%$ phosphorothioated siRNA, (c) 100\% phosphorothioated siRNA and (d) mRNA. The phosphate of the lipids is in plot (a-d) and for the cargo in (c-e), as well as their simulation using the spin diffusion model I (black trace). Simulated build-ups with $\mu$ waves on (black trace) and off (blue trace) are observed for phosphate lipids in 1 (b), 50\% (e) or 100\% phosphorothioated $\mathbf{2}$ and $\mathbf{3}$ as well as for the cargoes (c and f), respectively, are overlaid over the experimental ${ }^{31} \mathrm{P}$ saturation recovery data for $\mu$ waves on (blue) and off (black). Also, the $T_{\mathrm{B}}$ values reported on the plots are result from an analytical fit using a stretched exponential. 


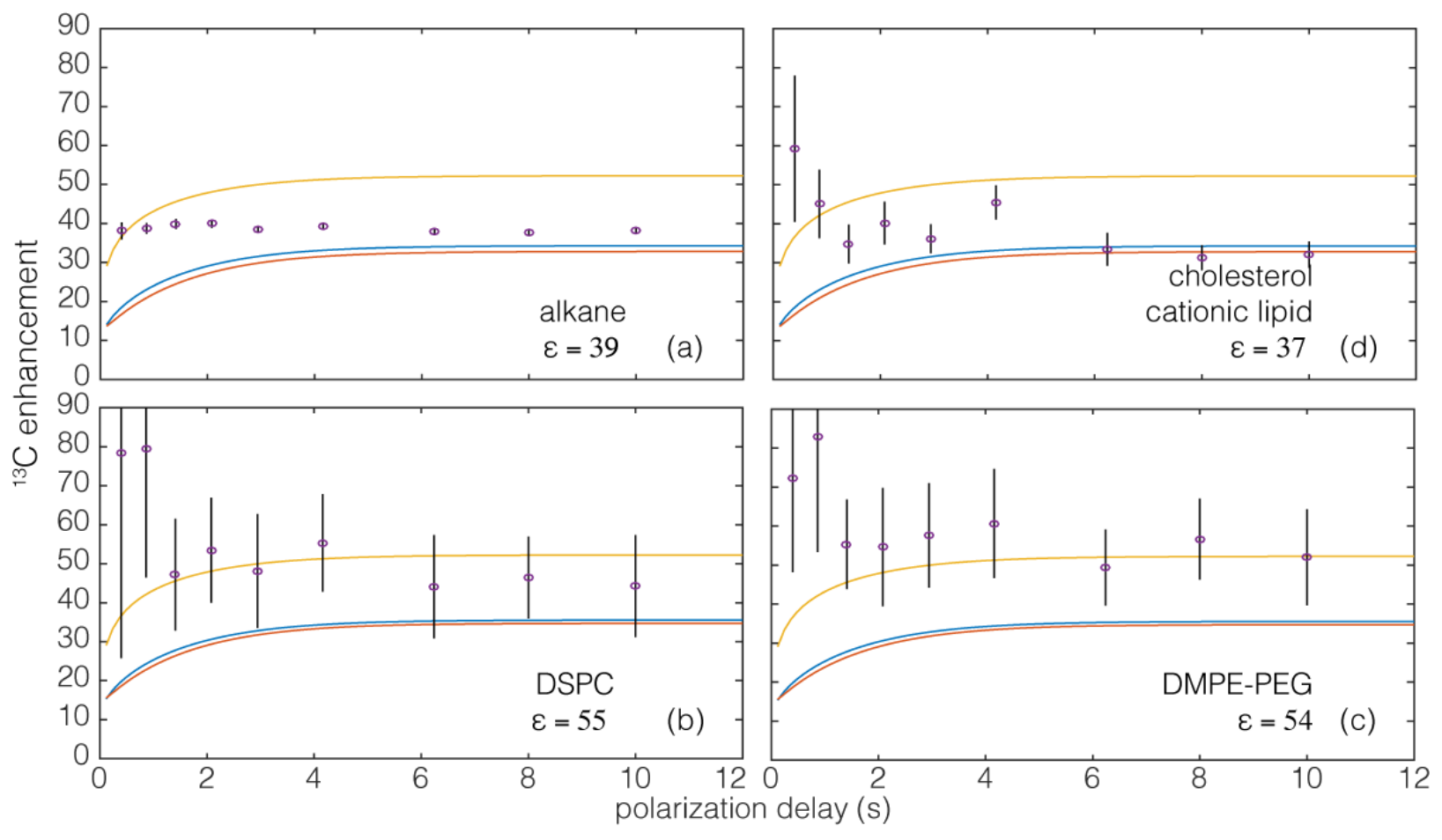

Figure S12. (a) Experimental plots of the enhancement $\left({ }^{13} \mathrm{C}\right)$ values as a function of polarization delay (s) for empty LNP chemical shifts at (a) $30 \mathrm{ppm}$, (b) $57 \mathrm{ppm}$, (c) $69 \mathrm{ppm}$ (d) $128 \mathrm{ppm}$. The specified enhancements on the plots is the average value. Their respective ${ }^{1} \mathrm{H}-{ }^{1} \mathrm{H}$ spin diffusion modeling using the layer model II are overlaid (colored traces: yellow for the surface layer; red for the core; blue surface layer and core average). 


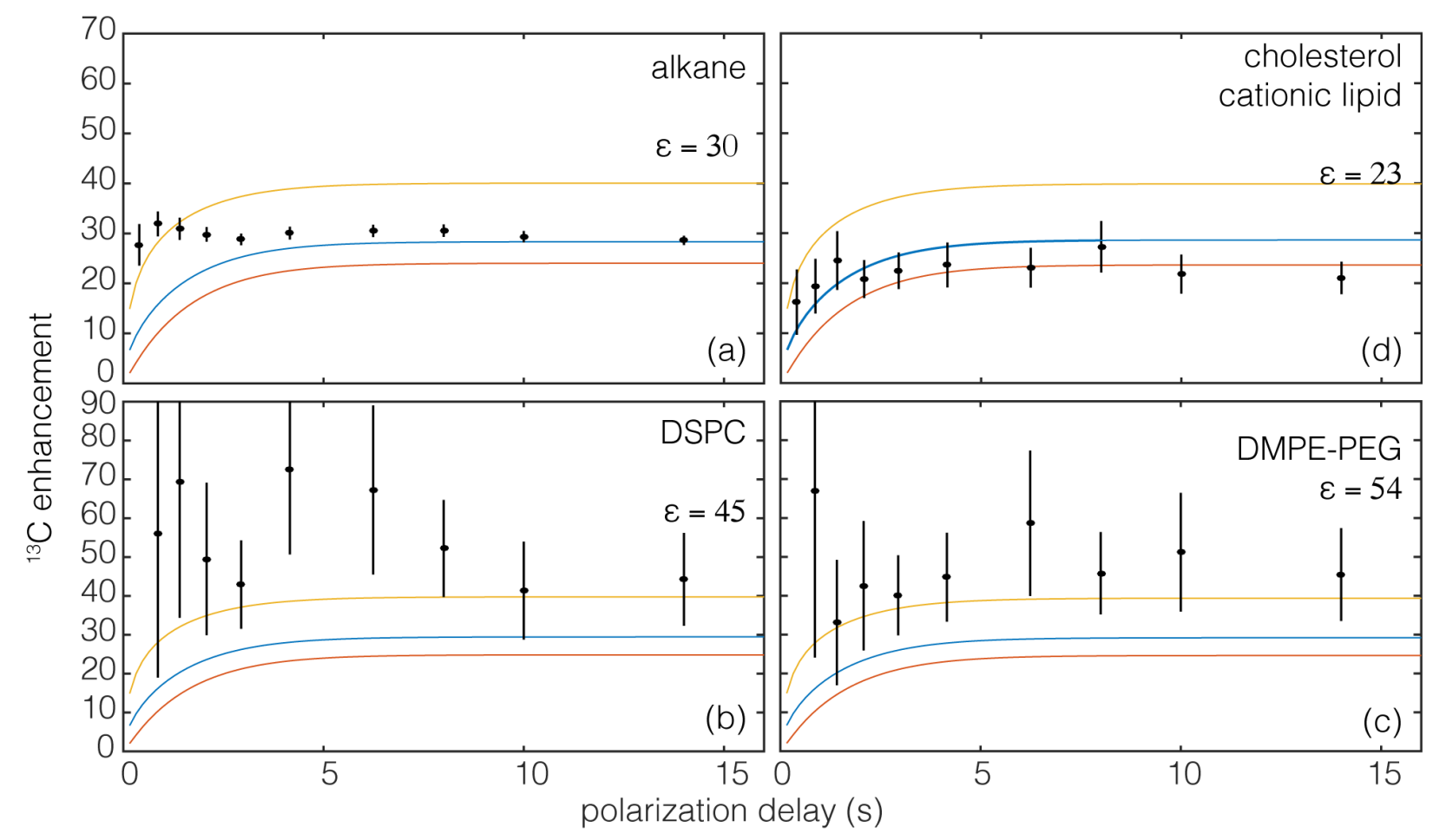

Figure S13. (a) Experimental plots of the enhancement $\left({ }^{13} \mathrm{C}\right)$ values as a function of polarization delay (s) for LNP encapsulated 100\% phosphorothioated siRNA at (a) $30 \mathrm{ppm}$, (b) $57 \mathrm{ppm}$, (c) 69 ppm (d) $128 \mathrm{ppm}$. The specified enhancements on the plots is the average value. Their respective ${ }^{1} \mathrm{H}-{ }^{1} \mathrm{H}$ spin diffusion modeling using the layer model II are overlaid (colored traces: yellow for the surface layer; red for the core; blue surface layer and core average). 


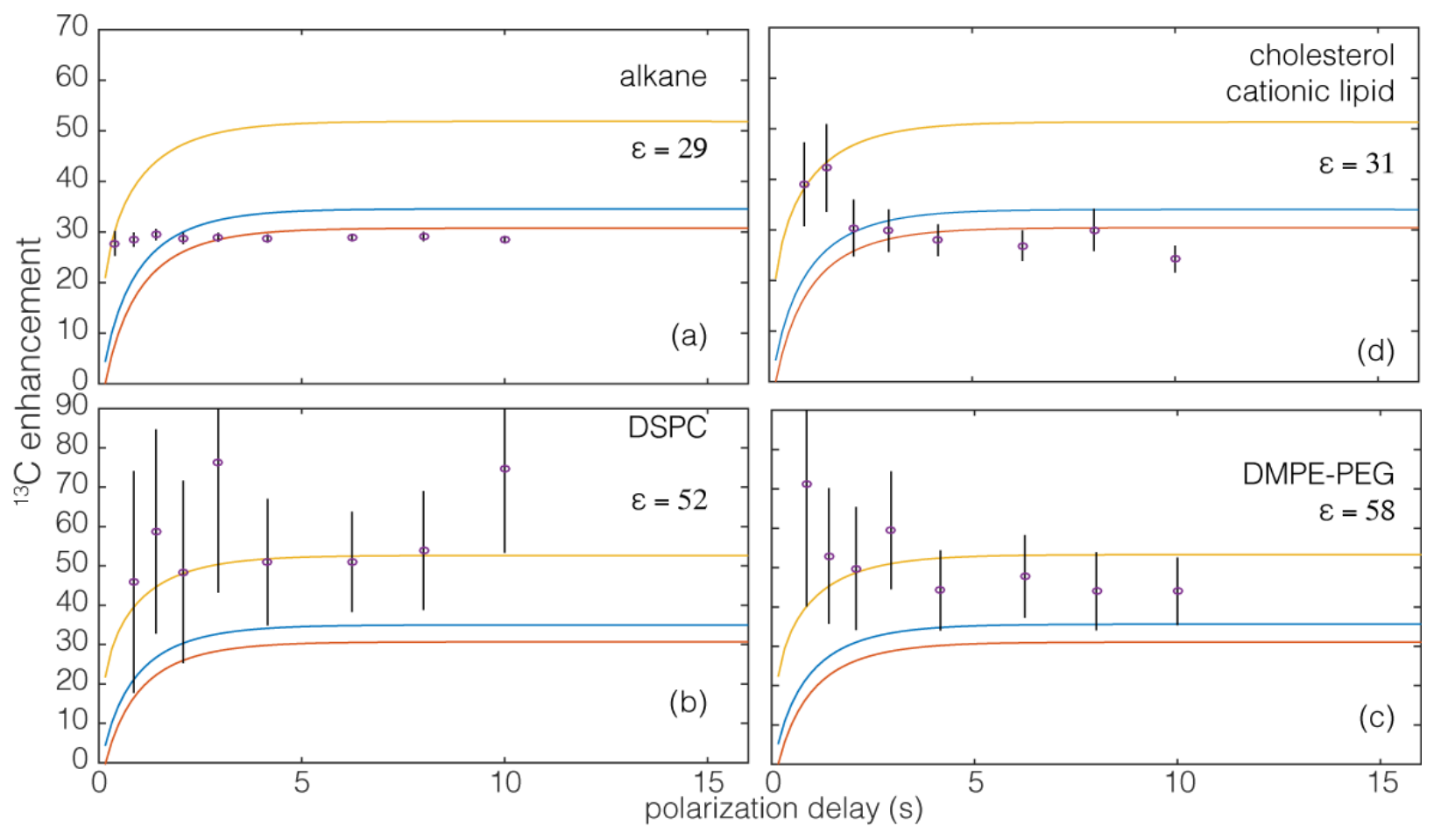

Figure S14. (a) Experimental plots of the enhancement $\left({ }^{13} \mathrm{C}\right)$ values as a function of polarization delay (s) for LNP encapsulated 50\% phosphorothioated siRNA chemical shifts at (a) 30 ppm, (b) $57 \mathrm{ppm}$, (c) $69 \mathrm{ppm}$ (d) $128 \mathrm{ppm}$. The specified enhancements on the plots is the average value. Their respective ${ }^{1} \mathrm{H}-{ }^{1} \mathrm{H}$ spin diffusion modeling using the layer model II are overlaid (colored traces: yellow for the surface layer; red for the core; blue surface layer and core average). 


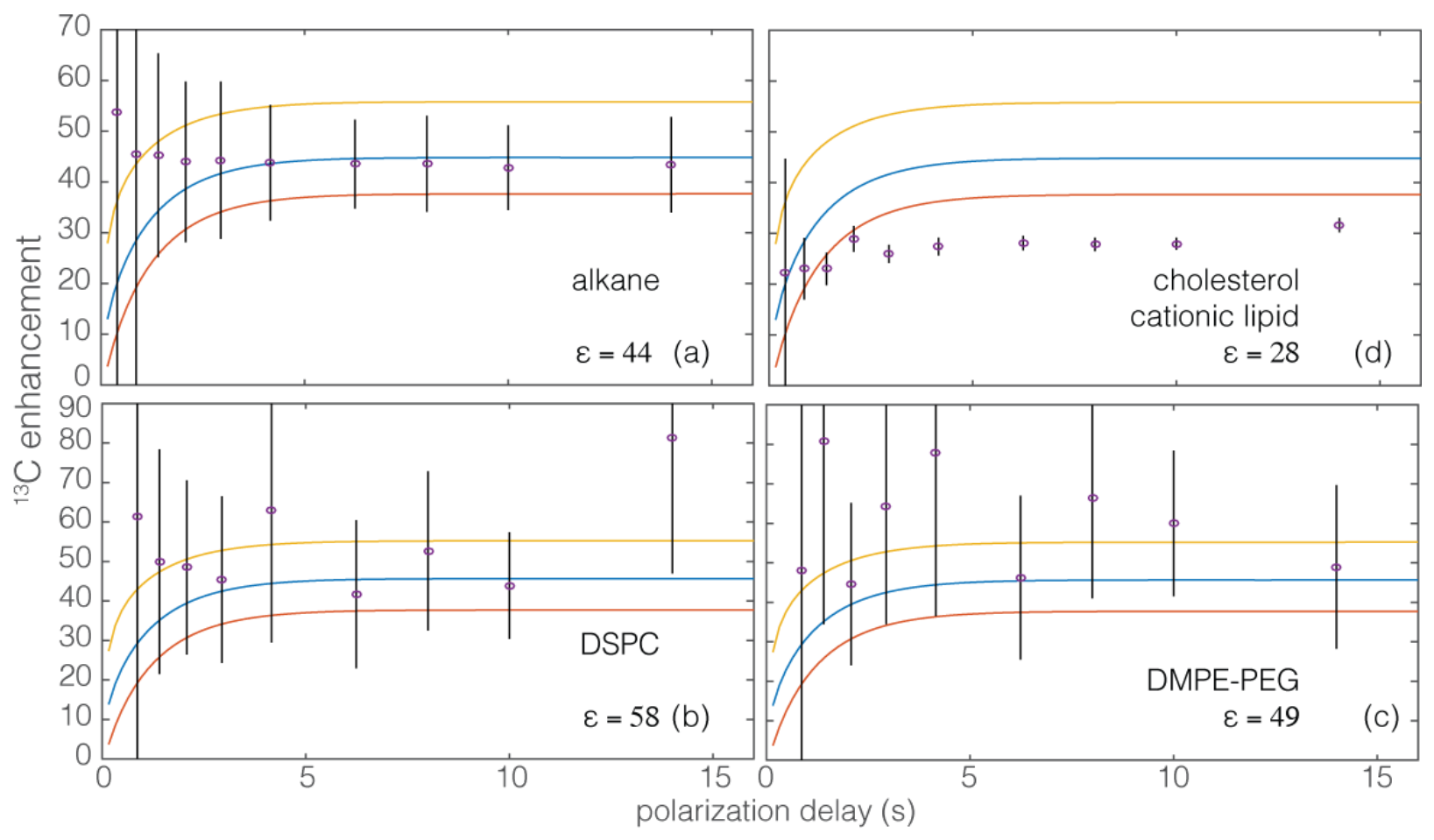

Figure S15. (a) Experimental plots of the enhancement $\left({ }^{31} \mathrm{C}\right)$ values as a function of polarization delay (s) for empty LNP, $\delta_{\text {iso }}\left({ }^{31} \mathrm{P}\right.$ ) at (a) $10 \mathrm{ppm}$, and LNP encapsulated siRNA chemical shifts at (b) $10 \mathrm{ppm}$, (c) $67 \mathrm{ppm}$. The specified enhancements on the plots is the average value. Their respective ${ }^{1} \mathrm{H}-{ }^{1} \mathrm{H}$ spin diffusion modeling using the layer model II are overlaid (colored traces: yellow for the surface layer; red for the core; blue surface layer and core average). 

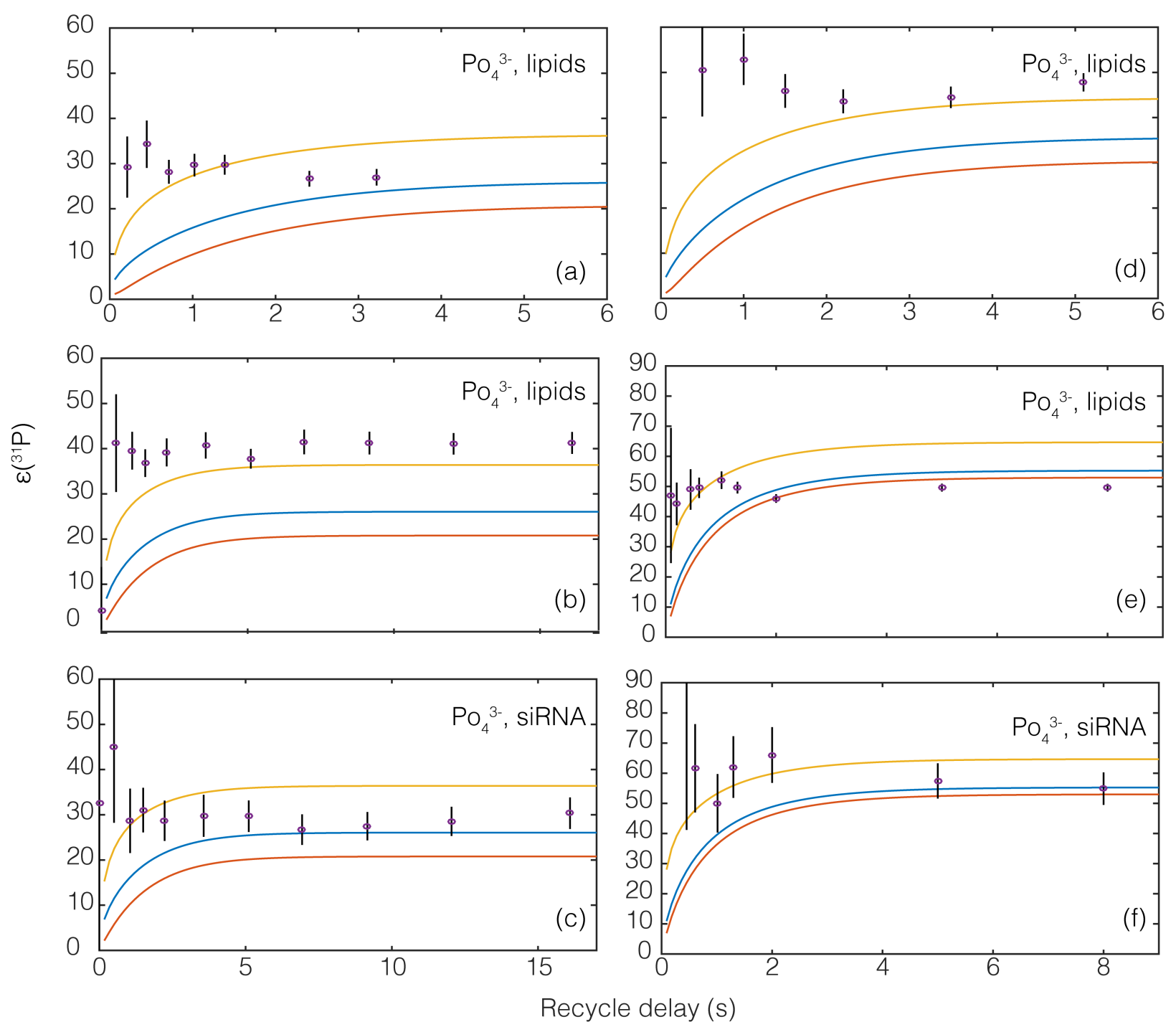

Figure S16. Experimental (purple circles) plots of the enhancement $\left({ }^{31} \mathrm{P}\right)$ values as a function of polarization delay for empty LNP (a) and in the presence of the cargo (b-c) 100\% phosphorothioated siRNA, LNP encapsulated mRNA (d) and LNP with (e-f) 50\% phosphorothioated siRNA.

\section{3."Back of the envelope" calculation of the volume inside LNPs}

As the DSPC is hydrophobic in nature, it will "escape" the internal water pool and become more surface rich. The probability of finding hydrophobic DSPC molecules at the surface of LNPs is verified by calculating the internal volume of LNPs that excludes its water content and associated electrostatic interaction radius. Below can be found the calculations and assumptions where, $\mathrm{r} 1$, 
r2, dr, are the radius for dry LNP (absence of water inside), LNP with $20 \%$ water inside, ${ }^{5}$ radius surrounding the water which corresponds to the assumption of electrostatic interactions used in COSMO solvation models, respectively. V1, V2, Vw and Ni correspond to the volumes for dry LNP (absence of water inside), the volume of LNP with $20 \%$ water inside, volume of the water and the number of water pools. MATLAB script to calculate the relationship between the internal LNP volume and number of water pools, and the plots are shown in Figure S17. The calculation shows a clear decrease in internal volume as a function of number of increasing water pools inside the LNP (Figure S15). This might justify our belief that DSPC becomes surface enriched when encapsulating hydrophilic cargoes as it has less volume to occupy inside the LNP.

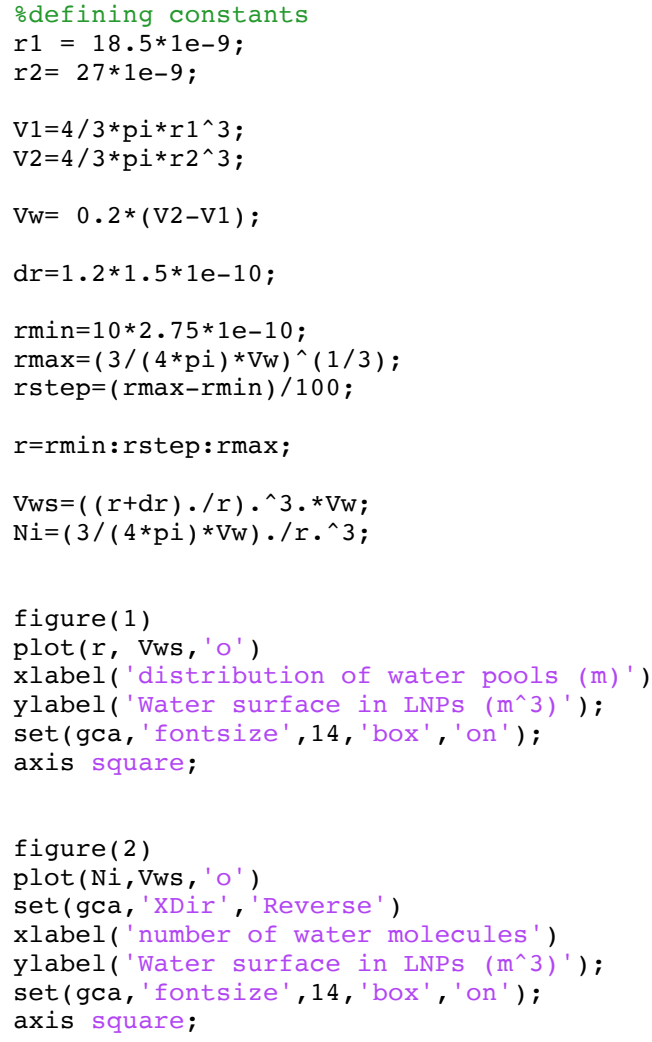




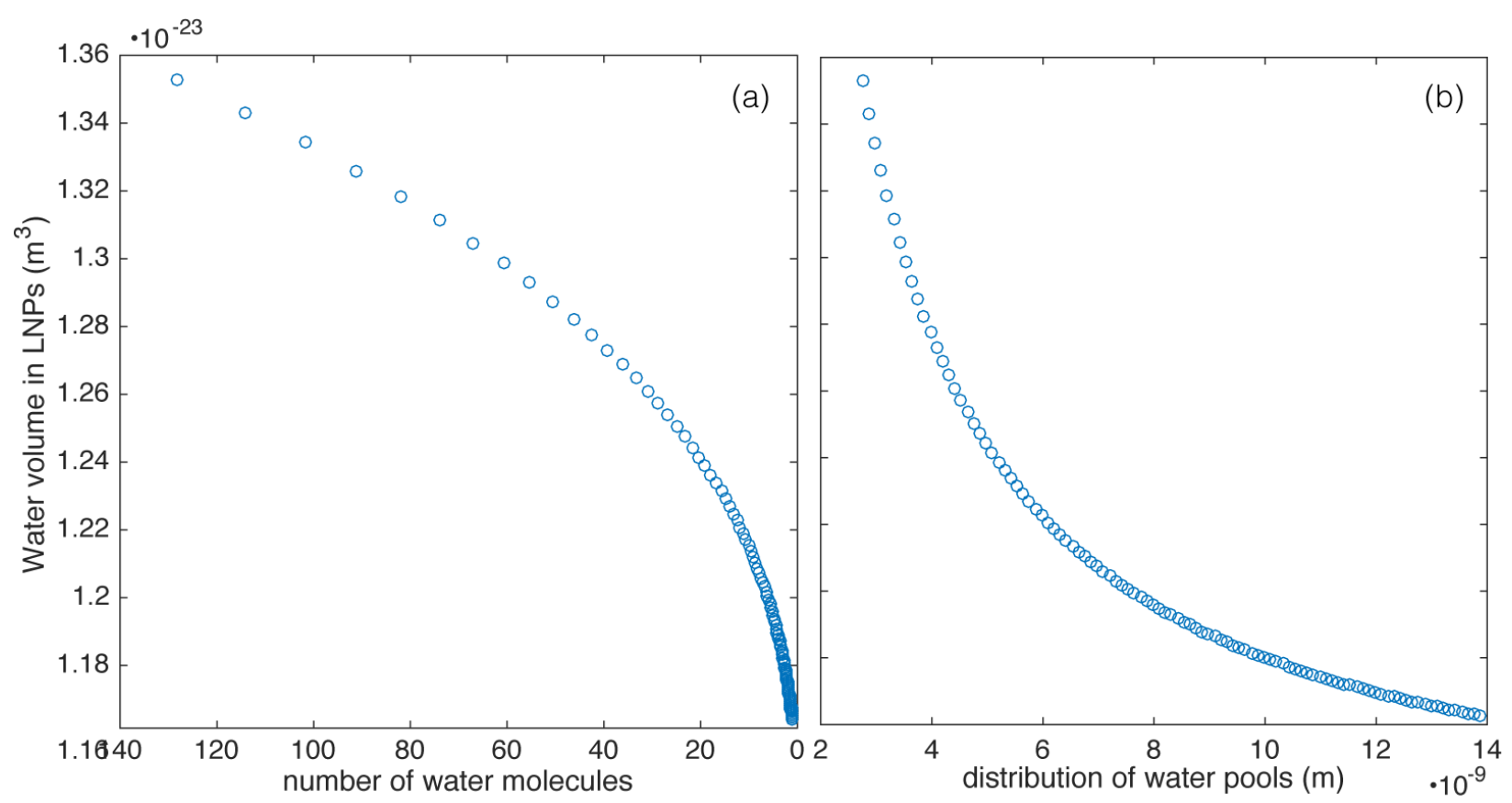

Figure S17. LNP volume as a function of decreasing internal water pools (a), and as a function of water pool size.

1. Zhigaltsev, I. V.; Belliveau, N.; Hafez, I.; Leung, A. K.; Huft, J.; Hansen, C.; Cullis, P. R., Bottom-up Design and Synthesis of Limit Size Lipid Nanoparticle Systems with Aqueous and Triglyceride Cores Using Millisecond Microfluidic Mixing. Langmuir 2012, 28, 3633-3640.

2. Kanasty, R.; Dorkin, J. R.; Vegas, A.; Anderson, D., Delivery Materials for Sirna Therapeutics. Nat. Mater. 2013, 12, 967-977.

3. Rossini, A. J.; Widdifield, C. M.; Zagdoun, A.; Lelli, M.; Schwarzwälder, M.; Copéret, C.; Lesage, A.; Emsley, L., Dynamic Nuclear Polarization Enhanced Nmr Spectroscopy for Pharmaceutical Formulations. J. Am. Chem. Soc. 2014, 136, 23242334.

4. Pinon, A. C., et al., Measuring Nano to Micro Structures from Relayed Dnp Nmr. The Journal of Physical Chemistry C 2017, 121, 15993-16005.

5. Leung, A. K.; Hafez, I. M.; Baoukina, S.; Belliveau, N. M.; Zhigaltsev, I. V.; Afshinmanesh, E.; Tieleman, D. P.; Hansen, C. L.; Hope, M. J.; Cullis, P. R., Lipid Nanoparticles Containing Sirna Synthesized by Microfluidic Mixing Exhibit an ElectronDense Nanostructured Core. J. Phys. Chem. C 2012, 116, 18440-18450. 